hep-th/0404039

UPR-T-1074, HIP-2004-TH/12

UCLA-04-TEP-20, ITFA-2004-11

\title{
String Scattering from Decaying Branes
}

\author{
Vijay Balasubramanian1, Esko Keski-Vakkuri], Per Kraus ${ }^{3}$ and Asad Naqvi \\ ${ }^{1}$ David Rittenhouse Laboratory, University of Pennsylvania, Philadelphia, PA 19104, USA \\ ${ }^{2}$ Helsinki Institute of Physics and Department of Physical Sciences, \\ P.O. Box 64, FIN-00014 University of Helsinki, Finland \\ ${ }^{3}$ Department of Physics, University of California, Los Angeles, 90095, USA \\ ${ }^{4}$ Institute for Theoretical Physics, University of Amsterdam, The Netherlands
}

\begin{abstract}
We develop the general formalism of string scattering from decaying D-branes in bosonic string theory. In worldsheet perturbation theory, amplitudes can be written as a sum of correlators in a grand canonical ensemble of unitary random matrix models, with time setting the fugacity. An approach employed in the past for computing amplitudes in this theory involves an unjustified analytic continuation from special integer momenta. We give an alternative formulation which is well-defined for general momenta. We study the emission of closed strings from a decaying D-brane with initial conditions perturbed by the addition of an open string vertex operator. Using an integral formula due to Selberg, the relevant amplitude is expressed in closed form in terms of zeta functions. Perturbing the initial state can suppress or enhance the emission of high energy closed strings for extended branes, but enhances it for D0-branes. The closed string two point function is expressed as a sum of Toeplitz determinants of certain hypergeometric functions. A large N limit theorem due to Szegö, and its extension due to Borodin and Okounkov, permits us to compute approximate results showing that previous naive analytic continuations amount to the large $\mathrm{N}$ approximation of the full result. We also give a free fermion formulation of scattering from decaying D-branes and describe the relation to a grand canonical ensemble for a $2 \mathrm{~d}$ Coulomb gas.
\end{abstract}

\footnotetext{
1 vijay@physics.upenn.edu

2 esko.keski-vakkuri@helsinki.fi

3 pkraus@physics.ucla.edu

4 anaqvi@science.uva.nl
} 


\section{Introduction}

D-branes, as solitons of open string theory that are localized in space, have given many insights into nonperturbative phenomena in string theory such as string duality, and resolve many timelike singularities of General Relativity including those inside some black holes. In addition, they lead to the holographic description of 11 dimensional asymptotically flat space via a Matrix model and of spaces with a negative cosmological constant in terms of a dual conformal field theory. Many of these developments, and particularly the last, arose from an understanding of D-brane dynamics - namely how closed strings scatter from such solitons which are quantized in terms of open string fluctuations.

It is of great interest to understand similar issues in the time dependent context of rapidly expanding universes, particularly in view of the likely occurrence of a Big Bang followed by inflation in the early universe and the possible presence of a positive cosmological constant $\Lambda>0$ now. Exploration of the symmetries and structure of universes with positive $\Lambda$ has suggested that if they have a holographic description the dual might be related to a Euclidean CFT living on the early or late time boundaries of such spacetimes ([1], [2]). D Time, in such a picture, emerges holographically via the RG flow of the Euclidean field theory dual in analogy with the emergence of the radial direction of AdS space from the RG flow of a Lorentzian field theory ([1], [2]). In order for such a picture to be actually realized in string theory one would need some kind of D-brane localized in time, called an S-brane in [3], with a decoupling limit relating the closed strings on the spacetime background to the open strings quantizing the brane. One might hope that the spacetime near such a Euclidean brane would be rapidly expanding by analogy with the rapid expansion of the transverse space in the vicinity of a conventional brane. In the decoupling limit for standard D-branes it is precisely this rapid transverse expansion that gives rise to the exponential increase in the volume of AdS spaces as the boundary is approached. Thus one might hope that a Euclidean brane would lead to a spacetime exponentially expanding in time in a suitable decoupling limit.

Sen has proposed [4] that S-branes are concretely realized in string theory by the exact boundary CFTs representing decaying branes. In bosonic string theory the exactly marginal boundary interaction

$$
S_{\mathrm{bndy}}=\lambda \int d t e^{X^{0}}
$$

(where $X^{0}$ is the timelike scalar) describes such a brane [5], [6], [7] 6 The basic results that have been obtained so far are:

1. At vanishing $g_{s}$ the brane decays to "tachyon matter" with energy but vanishing pressure 四.

5 One heuristic way of motivating this is to note that Lorentzian de Sitter space and Euclidean AdS space are solutions to $-X_{0}^{2}+X_{1}^{2}+\cdots X_{d}^{2}= \pm l^{2}$ embedded in flat $(d+1)$ dimensional space. Both hyperboloids are thus different real sections of the same complex manifold.

6 There are also cosh $X^{0}$ and $\sinh X^{0}$ interactions, which describe brane formation and subsequent decay [4]. Note the analogy with the inflationary and global parametrizations of de Sitter space, namely $d s^{2}=-d t^{2}+e^{t} d \vec{x}^{2}$ versus $d s^{2}=-d t^{2}+\cosh (t)^{2} d \Omega^{2}$. 
2. At finite coupling there is tremendous production of very heavy non-relativistic closed strings 8 .

3. It has been proposed that the resulting coherent state of heavy closed strings is an equivalent description of the tachyon matter.

4. In view of (2) and (3) it is proposed that there is a new open-closed duality hinting at a new kind of holography [9], [10].

5. There is a close relation with the dynamics of decaying unstable branes in the $c=1$ matrix model that suggests that the picture $(1)$ - (4) is essentially correct ([11], [12], [13, 14]).

To understand the structure of tachyon matter, and to explore the possibility of a decoupling limit leading to timelike holography, a central problem is to compute general scattering amplitudes of closed and open strings from the decaying brane. Closed strings can be used particularly to probe the structure of the tachyon matter final state that exists after the brane decays, and open strings, since they will only be present in the spectrum at early times, can be used to change the initial brane configuration which is decaying.

Thus, in Sec. 2 and 3 we develop the general formalism of string scattering from decaying branes in bosonic string theory. In a perturbative approach, amplitudes can be written as a sum of correlators in a grand canonical ensemble of unitary matrix models, with time setting the fugacity. If we integrate over the zero-mode time coordinate at the outset, the amplitudes are connected by analytic continuation to a single unitary matrix model, the rank of which is related to the total energy carried by the external vertex operators. Based on the approach which was employed with success in bulk Liouville theory [15], it was proposed in ([7], [16]) to compute bulk correlation functions by "analytically continuing" the vertex operator momenta from discrete integer values where direct computation is simple. This procedure has long been known to be somewhat questionable since an analytic function cannot be defined from just the data at a discrete set of points without additional constraints such as knowledge of the behavior at infinity or consistency conditions imposed by symmetries. In the case of Liouville theory, suitable consistency conditions were found (see the review [17]) and one can try to exploit the resulting techniques to explore the physics of decaying branes ([7], [18], [19], [20], [21]). For full branes, an interesting prescription to compute $n$-point closed string disk amplitudes was given in terms of viewing the brane as an array of ordinary D-branes in imaginary time [22] (for generalization to higher genus, see [23]). In this paper, we describe an alternative formulation of the scattering amplitude calculation which is well-defined in an open subset of the complex energy plane and therefore permits reliable analytic continuation. We will study in detail the open-closed and closed-closed two point functions, and compare our results to previous work.

In Sec. 4, we study the emission of closed strings from a decaying brane with initial conditions perturbed by the addition of an open string vertex operator. U Using an integral formula due to Selberg, the relevant amplitude is expressed in closed form in terms of zeta functions. Perturbing the initial state in this way can either suppress or enhance the emission of high energy closed strings for extended branes, but always enhances it for

7 Another approach to these amplitudes will be described in [21]. 
D0-branes. This is consistent with the picture that D0-branes decay entirely into closed strings, with very heavy closed strings making up the tachyon matter state, while higher dimensional branes can decay partly into unstable lower dimensional branes. However, the enhancement of emission that we find in some cases will increase the direct closed string emission even by the higher dimensional branes. We discuss the consequences for the hypothesis that tachyon matter is nothing but a state of very heavy closed strings. In Sec. 5 , the closed string two point function is expressed as a sum on $N$ of Toeplitz determinants of certain $N \times N$ matrices of hypergeometric functions. A large-N limit theorem due to Szegö, and its extension due to Borodin and Okounkov, permit us to compute approximate results that show that previously used methods to compute scattering amplitudes in the decaying brane amount to a leading large $N$ approximation from our perspective. In Appendix $\mathrm{C}$ we give a free fermion formulation of scattering from decaying branes by extending old techniques of Douglas [24]. In Appendix D we describe the relation of the decaying brane correlators to a grand canonical ensemble for a classical Coulomb gas.

\section{Review of closed string tachyon scattering from D-branes}

To establish notation and conventions, in this section we review the standard computation of closed string tachyon scattering from a D-brane. This amounts to computing the bulk two point function on the disk, with Neumann and Dirichlet boundary conditions in the various directions.

\subsection{Kinematics}

We follow the conventions of Polchinski [25], with $\eta^{\mu \nu}=(-,+,+,+, \ldots)$, and $\alpha^{\prime}=1$. The closed string tachyon vertex operator is

$$
e^{i k \cdot X}, \quad k^{2}=-m^{2}=4
$$

For scattering from a Dp-brane, we divide the momenta into the $p+1$ parallel components $k^{\|}$, and the $25-p$ transverse components $k^{\perp}$. We write the momenta of the two closed string tachyons as $k_{1}=\left(k^{\|}, k_{1}^{\perp}\right)$ and $k_{2}=\left(-k^{\|}, k_{2}^{\perp}\right)$. The Mandelstam variables are defined as

$$
s=2\left(k^{\|}\right)^{2}, \quad t=k_{1} \cdot k_{2} .
$$

We can factorize the amplitude in the closed or open string channels, and find poles at the location of physical string states:

$$
\begin{array}{cl}
\text { closed }: & k^{2}=-m^{2}=-4(N-1), \\
\text { open }: & k^{2}=-m^{2}=-(N-1)
\end{array}
$$

with $N=0,1,2, \ldots$. This implies that poles occur at

$$
\begin{gathered}
\text { closed : } t=-2(N+1) \\
\text { open : } s=-2(N-1) .
\end{gathered}
$$




\subsection{Correlators on disk}

We work on the unit disk, $|z| \leq 1$. As is standard, we separate out the zero modes from the worldsheet fields $X^{\mu}(z, \bar{z})$ by writing

$$
X^{\mu}(z, \bar{z})=x^{\mu}+X^{\prime} \mu(z, \bar{z})
$$

with $\int d^{2} z X^{\prime} \mu(z, \bar{z})=0$. The zero mode integrals are done at the end of the calculation, and enforce momentum conservation in Neumann directions.

The Neumann and Dirichlet correlators on the disk are

$$
\left\langle X^{\prime \mu}(z, \bar{z}) X^{\prime \nu}(w, \bar{w})\right\rangle= \begin{cases}-\frac{1}{2} \eta^{\mu \nu}\left[\ln |z-w|^{2}+\ln |1-z \bar{w}|^{2}\right] & N \\ -\frac{1}{2} \eta^{\mu \nu}\left[\ln |z-w|^{2}-\ln |1-z \bar{w}|^{2}\right] & D .\end{cases}
$$

For $z$ in the bulk and $w$ on the boundary $\left(w=e^{i t}\right)$ :

$$
\left\langle X^{\prime} \mu(z, \bar{z}) X^{\prime} \nu(t)\right\rangle=\left\{\begin{array}{cc}
-\eta^{\mu \nu} \ln \left|z-e^{i t}\right|^{2} & N \\
0 & D .
\end{array}\right.
$$

For both points on the boundary:

$$
\left\langle X^{\prime} \mu\left(t_{1}\right) X^{\prime} \nu\left(t_{2}\right)\right\rangle=\left\{\begin{array}{cc}
-\eta^{\mu \nu} \ln \left|e^{i t_{1}}-e^{i t_{2}}\right|^{2} & N \\
0 & D .
\end{array}\right.
$$

For self-contractions in the bulk:

$$
\left\langle X^{\prime \mu}(z, \bar{z}) X^{\prime \nu}(z, \bar{z})\right\rangle=\left\{\begin{array}{cc}
-\frac{1}{2} \eta^{\mu \nu} \ln |1-z \bar{z}|^{2} & N \\
\frac{1}{2} \eta^{\mu \nu} \ln |1-z \bar{z}|^{2} & D .
\end{array}\right.
$$

For self-contractions on the boundary:

$$
\left\langle X^{\prime \mu}(t) X^{\prime} \nu(t)\right\rangle= \begin{cases}0 & N \\ 0 & D\end{cases}
$$

8 In fact, there is a subtlety in defining the Neumann correlator. The Green's function which obeys Neumann boundary conditions and vanishes when integrated over the disk is $-\frac{1}{2} \eta^{\mu \nu}\left[\ln |z-w|^{2}+\ln |1-z \bar{w}|^{2}-z \bar{z}-w \bar{w}-c\right]$, where $c$ is a number which depends on the choice of worldsheet metric. The extra terms drop out after using spacetime momentum conservation (see section 6.2 of [25]), and so don't contribute to scattering from an ordinary D-brane. However, such terms do seem to contribute when considering D-branes with nontrivial worldvolume fields, as is our interest. Nevertheless, following standard practice, we will continue to drop these terms by adopting this as our prescription for defining the (naively divergent) $X^{0}$ path integral. 


\subsection{Closed string tachyon scattering from static D-brane}

Using the above correlators, the two point function of bulk exponentials is

$$
\left\langle e^{i k_{1} \cdot X^{\prime}(z, \bar{z})} e^{i k_{2} \cdot X^{\prime}(w, \bar{w})}\right\rangle=|z-w|^{t}|1-z \bar{w}|^{-s-t}|1-z \bar{z}|^{\frac{s}{2}-2}|1-w \bar{w}|^{\frac{s}{2}-2} .
$$

The S-matrix amplitude is given by fixing one vertex operator at the origin, $z=0$, and integrating $w$ over the disk. Up to an overall constant and a momentum conserving delta function, the amplitude is

$$
\int_{0}^{1} d r r r^{t}\left(1-r^{2}\right)^{\frac{s}{2}-2}=\frac{\Gamma\left(\frac{t}{2}+1\right) \Gamma\left(\frac{s}{2}-1\right)}{\Gamma\left(\frac{s}{2}+\frac{t}{2}\right)},
$$

which indeed exhibits poles in accord with (2.4).

In the remainder of this work we are interested in computing the scattering amplitude from a decaying D-brane. Physically, as a function of $x^{0}$, we expect to find an amplitude which interpolates between that of a D-brane and that of a collection of closed strings (or tachyon matter) into which the D-brane decays. One signature of this is that the open string poles should be absent at late times. Besides this, it is difficult to exhibit any "smoking gun" signatures of the presence/absence of the D-brane. For instance, it is known that in the high energy and fixed angle regime, both types of amplitudes behave as $e^{-E^{2} f(\theta)}$, indicating softness at short distance.

\section{Scattering from the rolling tachyon}

Now we consider the tachyon profile $T\left(X^{0}\right)=\lambda e^{X^{0}}$ corresponding to the boundary interaction

$$
e^{-S_{\text {bndy }}}=e^{-\lambda e^{x^{0}} \int d t e^{x^{\prime 0}}}
$$

where we have separated out the zero mode as in (2.5). Consider the scattering of $\ell$ bulk tachyons. The $X^{0}$ part of the computation involves

$$
\begin{aligned}
A_{\ell} & =\int D X^{0} e^{-S} \prod_{a=1}^{\ell} e^{i \omega_{a} X^{0}\left(z_{a}, \bar{z}_{a}\right)} \\
& =\int d x^{0} e^{i x^{0} \sum_{a} \omega_{a}}\left\langle e^{-\lambda e^{x^{0}} \int d t e^{X^{\prime} 0}} \prod_{a=1}^{\ell} e^{i \omega_{a} X^{\prime 0}\left(z_{a}, \bar{z}_{a}\right)}\right\rangle .
\end{aligned}
$$

The full amplitude also contains terms involving the spacelike $X^{i}$ that are the same as for a standard D-brane, as well as integrals over the locations of the vertex operators $z_{a}$. As we will describe below the bulk vertex operators can be moved to the boundary $(|z|=1)$, to compute amplitudes with arbitrary numbers of open string tachyon vertex operators. We can always choose a gauge in which our vertex operators (assuming they carry nonzero energy) contain no timelike oscillators [26]. Thus the interesting part of any correlation function (e.g., for closed string scattering from a brane with a perturbed initial state) involves interactions with the boundary tachyon that are summarized by (3.2). 


\subsection{Perturbative approach and matrix integral formulation}

One approach is to expand (3.2) in powers of the boundary interaction; i.e. as a power series in $\lambda$. The magnitude of $\lambda$ can be changed by shifting $x^{0}$, so truncating the power series is only sensible when considering scattering processes which are dominated at early times. In general, we must keep and sum the entire series. For the cases we consider, the sum will have a finite radius of convergence.

Expanding, we obtain

$$
A_{\ell}=\int d x^{0} e^{i x^{0} \sum_{a} \omega_{a}} \sum_{N=0}^{\infty} \frac{\left(-2 \pi \lambda e^{x^{0}}\right)^{N}}{N !} \int \prod_{i=1}^{N} \frac{d t_{i}}{2 \pi}\left\langle e^{X^{\prime} 0}\left(t_{1}\right) \ldots e^{X^{\prime 0}\left(t_{N}\right)} \prod_{a=1}^{\ell} e^{i \omega_{a} X^{\prime 0}\left(z_{a}, \bar{z}_{a}\right)}\right\rangle .
$$

By separating out the $x^{0}$ integral in this way we are isolating the contribution to the total scattering amplitude from the partially decayed state of brane that is present at any particular time. The late time contribution from $x^{0} \rightarrow \infty$ should isolate the effects of the tachyon matter final state to which the brane is supposed to decay.

To calculate the fixed $x^{0}$ contributions we now need to evaluate

$$
a_{\ell}^{(N)}=\int \prod_{i=1}^{N} \frac{d t_{i}}{2 \pi}\left\langle e^{X^{\prime 0}\left(t_{1}\right)} \ldots e^{X^{\prime 0}\left(t_{N}\right)} \prod_{a=1}^{\ell} e^{i \omega_{a} X^{\prime} 0\left(z_{a}, \bar{z}_{a}\right)}\right\rangle .
$$

The Wick contractions are straightforwardly evaluated using the Green's functions in (2.6)(2.9), yielding

$$
\begin{aligned}
a_{\ell}^{(N)}= & {\left[\prod_{a<b}\left|z_{a}-z_{b}\right|^{-\omega_{a} \omega_{b}}\right]\left[\prod_{a b}\left|1-z_{a} \bar{z}_{b}\right|^{-\frac{1}{2} \omega_{a} \omega_{b}}\right] } \\
& \times \int \prod_{i=1}^{N} \frac{d t_{i}}{2 \pi}\left[\prod_{i<j}\left|e^{i t_{i}}-e^{i t_{j}}\right|^{2}\right]\left[\prod_{i a}\left|1-z_{a} e^{-i t_{i}}\right|^{2 i \omega_{a}}\right] .
\end{aligned}
$$

An elegant way of rewriting (3.5) is in terms of matrix integrals [6] [16]. In particular, the measure $\prod_{i=1}^{N} \frac{d t_{i}}{2 \pi} \prod_{i<j}\left|e^{i t_{i}}-e^{i t_{j}}\right|^{2}$ is nothing but the measure for $U(N)$ matrices expressed in the eigenvalue basis, the product of exponentials being the Vandermonde determinant. This leads to the identification

$$
a_{\ell}^{(N)}=N !\left[\prod_{a<b}\left|z_{a}-z_{b}\right|^{-\omega_{a} \omega_{b}}\right]\left[\prod_{a b}\left|1-z_{a} \bar{z}_{b}\right|^{-\frac{1}{2} \omega_{a} \omega_{b}}\right] \int_{U(N)} d U \prod_{a}\left|\operatorname{det}\left(1-z_{a} U\right)\right|^{2 i \omega_{a}}
$$

where is $U$ is a unitary $N \times N$ matrix, and we have normalized the measure to $\int_{U(N)} d U=1$.

We can now write

$$
A_{\ell}=\left[\prod_{a<b}\left|z_{a}-z_{b}\right|^{-\omega_{a} \omega_{b}}\right]\left[\prod_{a b}\left|1-z_{a} \bar{z}_{b}\right|^{-\frac{1}{2} \omega_{a} \omega_{b}}\right] \int d x^{0} e^{i x^{0} \sum_{a} \omega_{a}} F\left(z_{1}, \omega_{1}, \ldots, z_{\ell}, \omega_{\ell} ; \mu\right)
$$


where we have isolated the relevant summation by defining

$$
F\left(z_{1}, \omega_{1}, \ldots, z_{\ell}, \omega_{\ell} ; \mu\right)=\sum_{N=0}^{\infty} e^{-N \mu} \int_{U(N)} d U \prod_{a}\left|\operatorname{det}\left(1-z_{a} U\right)\right|^{2 i \omega_{a}}
$$

and

$$
\mu=-x^{0}-\ln (-2 \pi \lambda)
$$

Here $\mu$ plays the role of a complex chemical potential, controlling the weights of the various $U(N)$ matrix integrals. As we will discuss in Appendix D there is indeed a precise correspondence to the grand canonical ensemble of a statistical mechanical system. Note that the late time behavior corresponds to $\operatorname{Re}(\mu) \rightarrow-\infty$.

The essential problem is to compute the matrix integrals appearing in (3.8), perform the sum over $N$, and evaluate the $x^{0}$ integral. Finally, to compute a string theory S-matrix element, we must also integrate over the positions of the vertex operators.

Having obtained (3.7) for general bulk tachyon amplitudes, it is simple to include boundary tachyons. Simply take any number of vertex operators to the boundary, $z_{a} \rightarrow 1$, and remove the corresponding factors of $\left|1-z_{a} \bar{z}_{a}\right|^{-\frac{1}{2} \omega_{a}^{2}}$, since there are no boundary selfcontractions according to $(2.10)$.

\subsection{Integrated approach: performing the zero mode integral first}

An alternative approach is based on a strategy which was employed with success in bulk Liouville theory [15]. Instead of leaving the $x^{0}$ integration until the end, we can perform it at the outset. This approach requires analytic continuation in the momenta and can miss non-analytic pieces that are known to be present in the amplitude. Nevertheless, useful information can be obtained.

So let us return to (3.2) and perform the $x^{0}$ integral using

$$
\int_{-\infty}^{\infty} d x^{0} e^{-\alpha e^{x^{0}}} e^{i \omega x^{0}}=\alpha^{-i \omega} \Gamma(i \omega)
$$

to obtain

$$
A_{\ell}^{\mathrm{int}}=(2 \pi \lambda)^{-i \sum_{a} \omega_{a}} \Gamma\left(i \sum_{a} \omega_{a}\right)\left\langle\left[\int \frac{d t}{2 \pi} e^{X^{\prime} 0}(t)\right]^{-i \sum_{a} \omega_{a}} \prod_{a=1}^{\ell} e^{i \omega_{a} X^{\prime 0}\left(z_{a}, \bar{z}_{a}\right)}\right\rangle .
$$

Using the identity $\Gamma(1-i z) \Gamma(i z)=-i \pi / \sinh \pi z$ we can write this as

$$
\begin{aligned}
A_{\ell}^{\mathrm{int}} & =-i \pi \frac{(2 \pi \lambda)^{-i \sum_{a} \omega_{a}}}{\sinh \left(\pi \sum_{a} \omega_{a}\right)} B_{\ell} \\
B_{\ell} & =\frac{1}{\Gamma\left(1-i \sum_{a} \omega_{a}\right)}\left\langle\left[\int \frac{d t}{2 \pi} e^{X^{\prime 0}(t)}\right]^{-i \sum_{a} \omega_{a}} \prod_{a=1}^{\ell} e^{i \omega_{a} X^{\prime 0}\left(z_{a}, \bar{z}_{a}\right)}\right\rangle .
\end{aligned}
$$


As we will see, the overall factor in $A_{\ell}^{\text {int }}$ is of exactly the form expected for the closed string one-point function [8]. The expression for $B_{\ell}$ contains complex powers of the fields and therefore must be defined by appropriate analytic continuation. In particular, to apply standard techniques, we need $-i \sum_{a} \omega_{a}$ to be a positive integer. We will now follow the procedure of defining $B_{\ell}$ by continuing the external momenta to imaginary integer values and then "continuing" back. This procedure requires some care, since in general the values of a function at a discrete set of arguments does not determine its behavior in the entire complex plane even with an assumption of analyticity. However, we will find situations in which the expression for $B_{\ell}$, evaluated formally for imaginary integer momenta, is actually defined on some open subset of the complex $\omega$ plane, thus permitting reliable analytic continuation.

Proceeding in this fashion, we perform the continuation

$$
i \omega_{a} \rightarrow-n_{a}
$$

to non-negative integers $n_{a}$, finding

$$
B_{\ell}=\frac{1}{N !}\left\langle\left[\prod_{j=1}^{N} \int \frac{d t_{j}}{2 \pi} e^{X^{\prime 0}\left(t_{j}\right)}\right] \prod_{a=1}^{\ell} e^{-n_{a} X^{\prime 0}\left(z_{a}, \bar{z}_{a}\right)}\right\rangle
$$

with $N=\sum_{a} n_{a}$. The correlator is the same as in (3.4) after the substitution (3.13). The result is therefore

$$
B_{\ell}=\left[\prod_{a<b}\left|z_{a}-z_{b}\right|^{n_{a} n_{b}}\right]\left[\prod_{a b}\left|1-z_{a} \bar{z}_{b}\right|^{\frac{1}{2} n_{a} n_{b}}\right] \int_{U(N)} d U \prod_{a}\left|\operatorname{det}\left(1-z_{a} U\right)\right|^{-2 n_{a}} .
$$

Comparing with (3.7) an essential difference is that there is no longer a sum over $N$ which needs to be evaluated, and the exponents in (3.15) are restricted to special integer values (which we'll eventually have to continue to generic values).

At these imaginary integer momenta, and for sufficiently large $N$, the matrix integral (3.15) is $N$ independent, and can be efficiently evaluated group theoretically [16] or using $\mathrm{SU}(2)$ current algebra [7]. As shown in [16], for $N \geq \sum_{a} n_{a}$ (which applies to our case, since $\left.N=\sum_{a} n_{a}\right)$,

$$
\int d U \prod_{a}\left|\operatorname{det}\left(1-z_{a} U\right)\right|^{-2 n_{a}}=\prod_{a b}\left|1-z_{a} \bar{z}_{b}\right|^{-n_{a} n_{b}} .
$$

This then yields

$$
B_{\ell}=\prod_{a<b}\left|z_{a}-z_{b}\right|^{n_{a} n_{b}} \prod_{a b}\left|1-z_{a} \bar{z}_{b}\right|^{-\frac{1}{2} n_{a} n_{b}} .
$$

The final step is to "undo" the analytic continuation, by replacing $n_{a}=-i \omega_{a}$ in (3.17), which, as we have stressed, cannot be justified without additional information. Nevertheless, if we optimistically apply this procedure we are led to:

$$
A_{\ell}^{\text {int }}=-i \pi \frac{(2 \pi \lambda)^{-i \sum_{a} \omega_{a}}}{\sinh \left(\pi \sum_{a} \omega_{a}\right)} \prod_{a<b}\left|z_{a}-z_{b}\right|^{-\omega_{a} \omega_{b}} \prod_{a b}\left|1-z_{a} \bar{z}_{b}\right|^{\frac{1}{2} \omega_{a} \omega_{b}} .
$$


In Sec. 4 we will see that naively using (3.16) and then analytically continuing to real momenta leads to unphysical results for bulk-boundary amplitudes and we will describe a better procedure for computing $B_{\ell}$. In Sec. 5 we discuss different procedures for computing bulk-bulk amplitudes.

\subsection{Comparison of one-point functions}

It is helpful to compare the results for the tachyon one-point function $(\ell=1)$ obtained in the two approaches 9 By a conformal transformation we can take the bulk vertex operator to be at the origin of the disk, $z=0$. In the perturbative approach we find from (3.8) that $F=1 /\left(1+2 \pi \lambda e^{x^{0}}\right)$, and therefore

$$
A_{1}=\int d x^{0} \frac{e^{i \omega x^{0}}}{1+2 \pi \lambda e^{x^{0}}}=-i \pi \frac{(2 \pi \lambda)^{-i \omega}}{\sinh \pi \omega} .
$$

Comparing with (3.18) we then find an agreement between the two approaches: $A_{1}=A_{1}^{\text {int }}$.

Note that in the perturbative approach the sum over $N$ is convergent only for $\left|2 \pi \lambda e^{x^{0}}\right|<1$, but we define it in general by analytic continuation. The analytically continued sum vanishes exponentially for large $x^{0}$, which yields a convergent $x^{0}$ integral.

The finite radius of convergence of the sum over $N$ is a feature of all the amplitudes we have examined, and makes it challenging to extract the late time behavior. In particular, to find the late time behavior of the unintegrated amplitude we must first find the exact early time behavior, so that we have an exact formula to analytically continue. This in turn means that we must perform an exact summation of the perturbation series. In the calculation above this turned out to be easy, but the requisite sums become more challenging for higher point amplitudes. In light of this, it would be extremely useful to develop a method to access the late time behavior directly without resorting to analytic continuation.

Although we have so far focused on tachyon vertex operators, it is instructive to consider a couple of other examples. Using the perturbative approach we can compute the one-point function of $\partial X^{0} \bar{\partial} X^{0} e^{i \omega X^{0}}$ at the origin to be [6]

$$
\begin{aligned}
\left\langle e^{\left.-S_{\text {bndy }} \partial X^{0} \bar{\partial} X^{0} e^{i \omega X^{0}}\right\rangle}\right. & =\int d x^{0}\left[\frac{1}{2} \frac{e^{i \omega x^{0}}}{1+2 \pi \lambda e^{x^{0}}}-e^{i \omega x^{0}}\right] \\
& =-\frac{i \pi}{2} \frac{(2 \pi \lambda)^{-i \omega}}{\sinh \pi \omega}-2 \pi \delta(\omega) .
\end{aligned}
$$

The $\delta(\omega)$ terms comes from the $N=0$ term in the sum, which is special since the bulk fields have nothing to contract against. On the other hand, if we repeat this calculation in the approach where we first integrate over $x^{0}$ we will miss this $\delta(\omega)$ term. This is the because the latter approach is based on analytic continuation in $\omega$ and can therefore miss

9 This computation also applies to an arbitrary closed string state, since as pointed out in $\|$ ], we can always choose a gauge such that there are no timelike oscillators. 
non-analytic pieces. The $\delta(\omega)$ term can be shown to be responsible for energy conservation: it gives $T_{00}$ where $T_{\mu \nu}$ is the energy-momentum tensor of the decaying brane. The lesson is that the integrated approach can miss certain physically relevant non-analytic contributions, but can still be useful, provided we keep this lesson in mind.

Further subtleties are revealed when we study the one-point functions of higher dimension operators corresponding to massive string modes. A finite number of terms in the sum over $N$ will be "special", leading to integrands which diverge for late times as $e^{p x^{0}}$ for positive integer $p$ (this behavior was observed in [27], [28].) Such terms will also arise in amplitudes involving more than one bulk tachyon, since such operators will appear in the OPE. This behavior clearly makes the direct evaluation of $x^{0}$ integrals problematic. To define these integrals one should impose the constraints of conformal invariance, as explained in [29], [30]. In particular, after computing the tachyon one-point function, we can write the remaining operators as Virasoro descendants, and then use the fact that boundary state is conformally invariant to generate the remaining amplitudes.

\section{Bulk-boundary two-point function: Perturbing the initial brane state}

A two-point amplitude in which one of the bulk operators is moved to the boundary describes an open-closed scattering amplitude. The closed string could either be an instate or out-state, but because the D-brane is decaying and does not exist at late times, the open string vertex operator can only be describing an in-state. Thus such amplitudes provide a systematic way of exploring the effects of perturbations to the initial conditions describing a decaying brane. For example, we could construct a localized lump on the brane at early times and ask how long in time the effect of this deformation lasts.

In the previous sections, we have focused on closed string tachyons. However, the generalization to include an arbitrary closed string state is relatively straight-forward if we choose a gauge in which the on-shell closed string state with non-zero energy has no time-like oscillators [26]. In this gauge, the closed string vertex operator has the form

$$
V=e^{i \omega_{c} X^{0}} V_{s p}
$$

The spatial part of this operator $V_{s p}$ is constructed from the 25 spatial fields and is a Virasoro primary with conformal dimension $\Delta=1+\frac{\omega_{c}^{2}}{4}$. The computation of a bulkboundary amplitude factorizes into a product of the two point function in the time-like boundary Liouville theory and in the free spatial CFT. The form of the closed string vertex operator (4.1) is useful, because in the time-like boundary Liouville CFT the vertex operator has the same form as the tachyon vertex operator. In the spatial direction, the operator is non-trivial. We can write $V_{s p}$ as

$$
V_{s p}=\mathcal{P}\left(\partial \vec{X}, \partial^{2} \vec{X} \cdots, \bar{\partial} \vec{X}, \bar{\partial}^{2} \vec{X} \cdots\right) e^{i \vec{p} \cdot \vec{X}}
$$

for a polynomial $\mathcal{P}$. In computing the bulk-boundary two point function with such an arbitrary closed string state, the contribution from the spatial part of the CFT is given by

$$
\left\langle: \mathcal{P}\left(\partial \vec{X}, \partial^{2} \vec{X} \cdots, \bar{\partial} \vec{X}, \bar{\partial}^{2} \vec{X} \cdots\right) e^{i \vec{p} \cdot \vec{X}(0)}:: e^{i \vec{k} \vec{X}(t)}:\right\rangle
$$


where $t$ parameterizes the position of the open string vertex operator on the boundary. The integration over the zero modes in the Neumann directions will yield a factor of $\delta\left(\vec{p}_{\|}+\vec{k}_{\|}\right)$. This $\delta$-function will be multiplied by terms from the contractions of the bulk and boundary fields. For no open string vertex operator (or equivalently for $\vec{k}=0$ ) the result of these contractions is just a phase [8]. In particular, note then that the absolute square of the result is independent of $\omega_{c}$, even though $\omega_{c}$ enters into the definition of $V_{s p}$ via $\Delta=1+\frac{\omega_{c}^{2}}{4}$, a result which is most easily seen from the boundary state. In the case with the open string vertex operator there will be additional contributions from the various ways of contracting fields from the bulk and boundary operators. This result will in general depend on the open string momentum $\vec{k}$, and therefore on $\omega_{o}$. However, the squared result will again be independent of $\omega_{c}$ just as before: $\omega_{c}$ dependence can potentially enter only in the Dirichlet part of the computatation, but since the open string vertex operator has no momentum components in the Dirichlet directions, this part of the computation is identical to that without the open string vertex operator [8]. So as far as the spatial CFT is concerned, the effect of the open string vertex operator is to contribute a $\omega_{0}$ and $\mathcal{P}$ dependent factor, but not to alter the $\omega_{c}$ dependence. Since our primary interest is in the $\omega_{c}$ dependence, we will omit the extra $\omega_{0}$ and $\mathcal{P}$ dependence (though these could be straightforwardly computed), and so the formulas we will write below are strictly valid only for $\mathcal{P}=1$, i.e. for the closed string tachyon.

We now return to the computation of the bulk-boundary two point function in the time-like boundary Liouville theory. First consider the perturbative approach. To obtain a correlator with one bulk and one boundary operator from (3.7), we set $\ell=2$, use conformal invariance to place one operator at $z_{1}=0$ and the other at $z_{2}=1,10$ and discard the divergent factor $\left(1-z_{2} \bar{z}_{2}\right)^{-\frac{1}{2} \omega_{2}^{2}}$ since it is removed by boundary normal ordering. This gives

$$
A_{2}\left(\omega_{c}, \omega_{o}\right)=\int d x^{0} e^{i\left(\omega_{c}+\omega_{o}\right) x^{0}} F\left(0, \omega_{c}, 1, \omega_{o} ; \mu\right)
$$

with

$$
F\left(0, \omega_{c}, 1, \omega_{o} ; \mu\right)=\sum_{N=0}^{\infty} e^{-N \mu} I_{N}\left(\omega_{o}\right)
$$

and

$$
I_{N}\left(\omega_{o}\right)=\int_{U(N)} d U|\operatorname{det}(1-U)|^{2 i \omega_{o}} .
$$

Here $\mu$ is the "chemical potential" defined in (3.9) and $\omega_{c, o}$ are the energies of the closed and open string vertex operators.

Now consider the integrated approach. Following (3.12), the integrated amplitude is

$$
A_{2}^{\mathrm{int}}\left(\omega_{c}, \omega_{0}\right)=-i \pi \frac{(2 \pi \lambda)^{-i\left(\omega_{c}+\omega_{o}\right)}}{\sinh \left(\pi\left(\omega_{c}+\omega_{o}\right)\right.} B_{2}\left(\omega_{c}, \omega_{o}\right) .
$$

10 Actually, this step is not entirely innocent. Separating out the zero mode $x^{0}$ breaks conformal invariance, and so conformal invariance can only be restored after performing the $x^{0}$ integration. Since $x^{0}$ is noncompact, there can be subtleties associated with boundary terms, as discussed in [31]. 
Here $B_{2}$ is defined by "analytic continuation" from imaginary momenta. Setting $\omega_{c, o}=$ $i n_{c, o}$ gives

$$
B_{2}\left(i n_{c}, i n_{o}\right)=I_{N}\left(i n_{o}\right),
$$

with $N=n_{c}+n_{o}$. Below we will evaluate $A_{2}^{\text {int }}$ using both the integrated expression (4.7) and the perturbative expansion (4.4) and show that they agree. This bulk-boundary amplitude captures the main features of more general amplitudes, but in a cleaner context. It allows one to vary the initial state of the brane by creating an open string perturbation. We can therefore explore a key physical question: are the general features of brane decay sensitive to the precise initial state introduced by Sen. For these reasons, we turn to a detailed study of the bulk-boundary two point function.

\subsection{Use of the Selberg integral}

Our basic technical goal is to evaluate integrals of the form (4.6). This can be done with the help of a famous integral due to Selberg. (For a pedagogical review, see [32].) Following Sec. 2.1 of 33

$$
\begin{aligned}
\int d U|\operatorname{det}(1-U)|^{-2 \alpha} & =\frac{1}{N !} \int \frac{d t_{1}}{2 \pi} \cdots \frac{d t_{N}}{2 \pi} \prod_{i<j}\left|e^{i t_{i}}-e^{i t_{j}}\right|^{2} \prod_{i}\left|1-e^{-i t_{i}}\right|^{-2 \alpha} \\
& =\frac{2^{N^{2}-2 \alpha N}}{N !(2 \pi)^{N}} \int_{-\infty}^{\infty} \cdots \int_{-\infty}^{\infty} d x_{1} \cdots d x_{N} \prod_{i<j}\left|x_{i}-x_{j}\right|^{2} \prod_{l=1}^{N}\left(1+x_{l}^{2}\right)^{-N+\alpha}
\end{aligned}
$$

The Selberg integral of interest to us is [32]

$$
\int_{-\infty}^{\infty} d x_{1} \cdots d x_{N} \prod_{i<j}\left|x_{i}-x_{j}\right|^{2} \prod_{l=1}^{N}\left(1+x_{l}^{2}\right)^{-N+\alpha}=\frac{(2 \pi)^{N} N !}{2^{N^{2}-2 \alpha N}} \prod_{j=1}^{N} \frac{\Gamma(j) \Gamma(j-2 \alpha)}{(\Gamma(j-\alpha))^{2}} .
$$

We thus find that the integral relevant for the open-closed amplitude is

$$
I_{N}(i \alpha)=\int d U|\operatorname{det}(1-U)|^{-2 \alpha}=\prod_{j=1}^{N} \frac{\Gamma(j) \Gamma(j-2 \alpha)}{(\Gamma(j-\alpha))^{2}} .
$$

Selberg's integral converges for real $\alpha$ when $\alpha<\frac{1}{2}$, which is also the condition for the Gamma functions on the right side to have positive arguments. We will define the result for arbitrary $\alpha$ by analytic continuation from the convergent region. In order to define the integrated amplitudes (4.7) for general real momenta we will have to also analytically continue $N$. The latter seems particularly problematic since the left hand side of (4.11) is an integral over $U(N)$ matrices and the right side contains a discrete product involving $N$. tion,

Happily, progress can be made using the integral representation of the Gamma func-

$$
\log \Gamma(z)=\int_{0}^{\infty} \frac{d t}{t}\left[(z-1) e^{-t}-\frac{e^{-t}-e^{-z t}}{1-e^{-t}}\right],
$$


which is valid for $z>0$. (This domain of validity is the same as that required for the convergence of the Selberg integral (4.10), namely $\alpha<1 / 2$ for real $\alpha$.) Applying this identity gives the result:

$$
\log \left[I_{N}(i \alpha)\right]=\log \left[B_{2}(i(N-\alpha), i \alpha)\right]=\int_{0}^{\infty} \frac{d t}{t}\left(1-e^{\alpha t}\right)^{2} \frac{e^{-N t}-1}{2(1-\cosh t)} .
$$

Both $\alpha$ and $N$ can be analytically continued in this expression. We will use this below to compute and compare the perturbative (4.5) and integrated (4.7) 2-point scattering amplitudes. Although the integral expression is itself only convergent in some regions of the complex momentum plane, we will see that it can be computed exactly in terms of special functions in these regions which can then be continued to general values of the momenta.

\subsection{Computing the Bulk-boundary amplitude}

- Integrated approach: In the approach in which the zero mode is integrated at the outset we wish to evaluate (4.7). We can do this by analytically continuing (4.13) as $\alpha \rightarrow-i \omega_{o}$ and $N \rightarrow-i\left(\omega_{c}+\omega_{o}\right)$. This gives:

$$
\begin{aligned}
A_{2}^{\mathrm{int}}\left(\omega_{c}, \omega_{o}\right) & =-i \pi \frac{(2 \pi \lambda)^{-i\left(\omega_{c}+\omega_{o}\right)}}{\sinh \left(\pi\left(\omega_{c}+\omega_{o}\right)\right)} e^{\int_{0}^{\infty} d t H\left(t, \omega_{o}\right)\left(e^{i\left(\omega_{c}+\omega_{o}\right) t}-1\right)} \\
H\left(t, \omega_{o}\right) & \equiv \frac{\left(1-e^{-i \omega_{o} t}\right)^{2}}{2 t(1-\cosh t)}
\end{aligned}
$$

We will evaluate the integral in the exponent in closed form in terms of special functions below. But first let us compare (4.14) to the perturbative result (4.5).

- Perturbative calculation: In the perturbative approach we must calculate the sum in (4.5). In terms of the function $H\left(t, \omega_{o}\right)$ defined above, this becomes

$$
\begin{aligned}
F\left(0, \omega_{c}, 1, \omega_{0} ; \mu\right) & =\sum_{N=0}^{\infty} e^{-N \mu} e^{\int_{0}^{\infty} d t H\left(t, \omega_{o}\right)\left(e^{-t N}-1\right)} \\
& =\sum_{l, N=0}^{\infty} \frac{e^{-N \mu}}{l !} \int_{0}^{\infty} \prod_{a=1}^{l} d t_{a} H\left(t_{a}, \omega_{o}\right)\left(e^{-N t_{a}}-1\right)
\end{aligned}
$$

We can now expand out the product of $\left(e^{-t_{a} N}-1\right)$ factors in a series and explicitly carry out the sum on $N$. This gives

$$
F\left(0, \omega_{c}, 1, \omega_{o} ; \mu\right)=\sum_{l=0}^{\infty} \sum_{m=0}^{l} \frac{(-1)^{l-m}}{l !}\left(\begin{array}{c}
l \\
m
\end{array}\right) \int_{0}^{\infty} \prod_{a=1}^{l} d t_{a} H\left(t_{a}, \omega_{o}\right) \frac{1}{1+2 \pi \lambda e^{x^{0}-\sum_{b=1}^{m} t_{b}}}
$$

where we have substituted back for the chemical potential $\mu$ in terms of the coupling $\lambda$ and the zero mode $x^{0}$. The full amplitude (4.4) involves a Fourier transform of $x^{0}$ as in (4.4). 
We recognize the required transform from the expression (3.19) used for the computation of the closed string one-point function and find

$$
\begin{aligned}
A_{2}\left(\omega_{c}, \omega_{o}\right)=-i \pi & \frac{(2 \pi \lambda)^{-i\left(\omega_{c}+\omega_{o}\right)}}{\sinh \left(\pi\left(\omega_{c}+\omega_{o}\right)\right)} \sum_{l=0}^{\infty} \sum_{m=0}^{l} \frac{(-1)^{l-m}}{l !}\left(\begin{array}{c}
l \\
m
\end{array}\right) \\
& \times \int_{0}^{\infty} \prod_{a=1}^{l} d t_{a} H\left(t_{a}, \omega_{o}\right) e^{i\left(\omega_{c}+\omega_{o}\right) \sum_{b=1}^{m} t_{b}} .
\end{aligned}
$$

Carrying out the sums we arrive at the final result

$$
A_{2}\left(\omega_{c}, \omega_{o}\right)=-i \pi \frac{(2 \pi \lambda)^{-i\left(\omega_{c}+\omega_{o}\right)}}{\sinh \left(\pi\left(\omega_{c}+\omega_{o}\right)\right)} e^{\int_{0}^{\infty} d t H\left(t, \omega_{o}\right)\left(e^{i\left(\omega_{c}+\omega_{o}\right) t}-1\right)} .
$$

This precisely matches the integrated amplitude (4.14).11 For future purposes, we define

$$
G\left(\omega_{c}, \omega_{o}\right) \equiv \int_{0}^{\infty} d t H\left(t, \omega_{o}\right)\left(e^{i\left(\omega_{c}+\omega_{o}\right) t}-1\right)
$$

as the exponent in the bulk-boundary amplitude.

The fact that the integrated and the perturbative approaches match exactly greatly increases our confidence in the methods developed here. The integrated approach is based on defining the expressions in (3.12) by first expanding them for integer momenta, evaluating the integrals and then interpreting the result as definition of the amplitude for general momenta. Such an "analytic continuation" is inherently problematic - without further specification of asymptotic conditions there exist many analytic functions which coincide with the one at hand at integer momenta. By contrast, the perturbative approach does not suffer from this failing and thus the agreement between (4.18) and (4.14) is very encouraging. Nevertheless, both approaches used here involve analytic continuation in the momenta and run the risk of missing non-analytic pieces. In particular, the perturbative approach assumed a well-defined Fourier transform and thus could miss non-analytic contributions to the amplitude associated with zero momentum processes. Despite this we will be able to extract useful insights into the structure of D-brane decay from (4.18).

\subsection{Comparison with other approaches}

As discussed in the previous section, one might have proposed an alternative approach to evaluating these amplitudes, by analytically continuing $\omega_{2}$ to imaginary integer values $\left(-i \omega_{2}=n \in Z^{+}\right)$, since in that case [16] shows that

$$
\int_{U(N)} d U|\operatorname{det}(1-z U)|^{-2 n}=(1-z \bar{z})^{-n^{2}}
$$

11 Related results in the Liouville approach appear in 19. 
This expression is valid for $n$ and $N$ nonnegative integers with $n \leq N$. Except for the trivial case $N=0$, the region where this formula is valid is disjoint from the region $\alpha<\frac{1}{2}$ where the Selberg integral was valid prior to analytic continuation in $\alpha$. To compare the two approaches, set $\alpha=n \in Z^{+}$in (4.11) and take $z=1$ in (4.20) to get the proposed answer for the bulk-boundary amplitude. We find the following two possible results

$$
\int d U|\operatorname{det}(1-U)|^{-2 n}=\left\{\begin{array}{l}
\prod_{j=1}^{N} \frac{\Gamma(j) \Gamma(j-2 n)}{(\Gamma(j-n))^{2}}=\text { finite } \\
(0)^{-n^{2}}=\infty
\end{array}\right.
$$

The first expression is obtained from the Selberg formula used in (4.11) while the second arises from (4.20) as derived in [16]. The Selberg result is finite for $N>2 n$ and vanishes for $N \leq 2 n$. By contrast, the second formula diverges for any $N$. The consequence of this is that after continuation back to real momenta, naive use of the latter expression from [16] leads to a vanishing amplitude for all momenta, while the Selberg formula will lead to a finite result.

This situation can be given a useful worldsheet interpretation. When a bulk vertex operator is taken towards the boundary to define a bulk-boundary amplitude, it will collide with operators from the boundary interaction. Therefore, we need to add counterterms to operators which are taken to the boundary in order to dress them appropriately in the presence of the interaction. The second formula in (4.21) corresponds, however, to a bare vertex operator, and so diverges. On the other hand, the analytic continuation used to define the Selberg integral is a convenient way of regulating and renormalizing the operator, and so gives a finite result. Of the two results displayed in (4.21), the finite Selberg result is the physically relevant one.

The summation over $N$ that was carried out in the perturbative approach, and which led to (4.18) for the final amplitude, became possible because the Fourier transform in the definition of $A_{2}$ (4.4) simplified the expressions. However, it can also be of interest to do the sum on $N$ in (4.5) without integrating over the zero mode $x^{0}$. As we discussed earlier, the contributions to the scattering amplitude from late times (large $x^{0}$ ) could shed light on the nature of the "tachyon matter" state to which the brane is supposed to decay. Doing the perturbative sum in (4.5) exactly for fixed small $x^{0}$ and then continuing to large $x^{0}$ turns out to be very difficult for arbitrary momenta. However, it is possible to make progress for special imaginary integer momenta. The relevant techniques and results are presented in Appendix A.

\subsection{Initial state perturbations and the decay of the brane}

Our result (4.14) (or (4.18)) for closed string emission from a decaying brane perturbed by an additional open string tachyon allows us to explore how the emission of closed strings is modified when the initial conditions for the brane are changed. For example, we might make a tachyon lump on the brane by superposing open string vertex operators and ask how this affects the decay.

Important questions to answer are whether all the energy in the brane decays into closed strings, and what is the distribution of these strings. Sen has argued that brane 
decay leads to a pressureless state of "tachyon matter" [4]. A computation of closed string emission from decaying branes by [8] (summarized in eq. (3.19) in Sec. 3) showed that at tree level the emission of closed strings is exponentially suppressed in their energy. For low dimension branes this nevertheless implies a divergent total emission, because of the Hagedorn growth in the density of states. This divergence was interpreted as indicating that all the energy of the decaying brane would be converted into closed strings, and that very heavy strings would dominate the decay products. Such heavy strings would have the stress tensor of pressureless dust, thus suggesting that they constitute the mysterious tachyon matter. It was also argued that near the endpoint of the decay, including backreaction would ensure that energy conservation was satisfied so that the emission of closed strings shuts off after all the energy in the original brane has been converted. This was confirmed in the $c=1$ matrix model, where it was also found that it is crucial to take into account the quantum mechanical nature of the decaying brane [12]. By contrast higher dimensional branes had a finite total emission of energy. The conventional wisdom states that in this case, small perturbations can also lead to decay into higher co-dimension branes [6] which would account for the "missing energy" in the decay into closed strings.

Our results can enable a systematic exploration of how changing the brane initial conditions affects the decay products. To initiate this study we will compute a closed form expression for the closed string emission amplitude (4.14) in terms of special functions and then extract the asymptotics of the decay for large closed string energies.

Before plunging into the calculation, let us note that there are two possibilities for what one might mean by "changing the brane initial conditions". One interpretation involves describing the brane by a boundary state; then to change the boundary state we can act with an exponentiated open string vertex operator (at least to first order in the operator; beyond that there will be corrections). This corresponds to changing the classical open string tachyon profile of the decaying brane. To first order in the perturbation, the total emission rate into closed strings will then be the sum of the original rate plus a correction due to the perturbation. Here we will focus on an alternative interpretation, in which we add a single quantum open string excitation on top of the classical background. The open-closed amplitude then corresponds to the amplitude for the incoming open string to disappear and for a closed string to be created.

To compute the bulk-boundary amplitude (4.14) in closed form we must evaluate an integral of the form

$$
G\left(\omega_{c}, \omega_{o}\right)=\int_{0}^{\infty} \frac{d t}{2 t}\left(1-e^{-i \omega_{o} t}\right)^{2} \frac{e^{-\beta t}-1}{(1-\cosh t)} \quad ; \quad \beta=-i\left(\omega_{c}+\omega_{o}\right) .
$$

Notice first that this integral vanishes if $\omega_{o}=0$, i.e., in the absence of any open string perturbation. In that case,

$$
A_{2}\left(\omega_{c}, 0\right)=-i \pi \frac{(2 \pi \lambda)^{-i \omega_{c}}}{\sinh \left(\pi \omega_{c}\right)}
$$

reproducing the known result in [8]. So the full result has the form of a modulation of the emission amplitude without an open string perturbation by a factor of $e^{G}$. It is also easy 
to argue that the real part of $G$ will be negative so that the modulating factor suppresses the amplitude.

The challenge in evaluating the integral (4.22) occurs because as $t \rightarrow 0$, cancellations between the terms in the numerator are needed to cancel the overall power of $t^{3}$ in the denominator in this limit, leading to a finite integral. With this in mind, to evaluate $G$ for general momenta, observe that $G$ also vanishes if $\beta=i\left(\omega_{c}+\omega_{o}\right)=0$. Because of this, we can write

$$
G\left(\omega_{c}, \omega_{o}\right)=\left.\int_{0}^{\beta} d \beta \frac{\partial G}{\partial \beta}\right|_{\omega_{o}} \equiv-\int_{0}^{\beta} d \beta \int_{0}^{\infty} \frac{d t}{2}\left(1-e^{-i \omega_{o} t}\right)^{2} \frac{e^{-\beta t}}{(1-\cosh t)} .
$$

After expanding $\left(1-e^{i \omega_{o} t}\right)^{2}$, each term in $\partial G / \partial \beta$ can be evaluated with the help of the integral formula

$$
\int_{0}^{\infty}(1-\cosh t)^{\nu} e^{-\gamma t} d t=e^{i \pi \nu} 2^{-\nu} \frac{\Gamma(\gamma-\nu) \Gamma(2 \nu+1)}{\Gamma(\gamma+\nu+1)} ; \quad \operatorname{Re} \nu>-\frac{1}{2}
$$

which is listed in [34] (vol. 1, p. 163). This expression diverges as $\nu \rightarrow-1$, but the linear combination

$$
-e^{-i \pi \nu} 2^{-\nu-1} \Gamma(2 \nu+1)\left\{\frac{\Gamma(\gamma-\nu)}{\Gamma(\gamma+\nu+1)}-2 \frac{\Gamma\left(\gamma-\nu+i \omega_{0}\right)}{\Gamma\left(\gamma+\nu+1+i \omega_{o}\right)}+\frac{\Gamma\left(\gamma-\nu+2 i \omega_{o}\right)}{\Gamma\left(\gamma+\nu+1+2 i \omega_{o}\right)}\right\}
$$

needed here is well-defined as $\nu \rightarrow-1$.

The limit $\nu \rightarrow-1$ gives $\partial G / \partial \beta$ in terms of derivatives of the Gamma function. The integral with respect to $\beta$ that remains can be evaluated by again using the integral representation (4.13). The result can be written in terms of the Hurwitz $\zeta$ function

$$
\zeta(s, z)=\frac{1}{\Gamma(s)} \int_{0}^{\infty} \frac{t^{s-1} e^{-z t}}{1-e^{-t}}=\sum_{n=0}^{\infty} \frac{1}{(n+z)^{s}}
$$

and $\zeta^{m, n}(s, z) \equiv \frac{\partial^{m+n} \zeta(s, z)}{\partial s^{m} \partial z^{n}}$. In order to summarize the results, we introduce the notation

$$
\mathcal{H}[F(x), a, b)] \equiv F(b+a)-2 F(b)+F(b-a) .
$$

Then, after using various $\zeta$ function identities, we find the result:

$$
\begin{aligned}
G\left(\omega_{c}, \omega_{o}\right) & =\mathcal{H}\left[\zeta^{(1,0)}(-1, x),-i \omega_{o}, i \omega_{o}\right]-\mathcal{H}\left[\zeta^{(1,0)}(-1, x),-i \omega_{o},-i \omega_{c}\right] \\
& +\mathcal{H}\left[x(\zeta(0, x)-\ln \Gamma(x)),-i \omega_{o}, i \omega_{o}\right]-\mathcal{H}\left[x(\zeta(0, x)-\ln \Gamma(x)),-i \omega_{o},-i \omega_{c}\right]
\end{aligned}
$$

Putting this expression back into (4.18) gives the general bulk-boundary amplitude in closed form in terms of zeta functions. This result can now used to extract many aspects of the physics of decaying branes. Here we will explore one question - how does including an open string perturbation change the asymptotics of brane decay into closed strings? 
To do this we must find the asymptotics of $G\left(\omega_{c}, \omega_{o}\right)$ for $\omega_{c} \gg \omega_{o}$. We will use the expressions

$$
\begin{aligned}
\zeta(0, x) & =\frac{1}{2}-x \\
\zeta^{(1,0)}(-1, a) & =\frac{B_{2}(a) \ln a}{2}-\frac{B_{2}(a)+a}{4}+\frac{B_{2}}{4}-\sum_{k=3}^{\infty} \frac{a^{-k+2} B_{k}}{(k-2)(k-1) k} .
\end{aligned}
$$

Here $B_{2}(a)=a^{2}-a+1 / 6$ and $B_{2}=1 / 6$ are the second Bernoulli polynomial and number respectively. The second formula was derived for $\Re(a) \rightarrow \infty$ [35], but it will apply by analytic continuation for large $|a|$ in general. Applying these formulae we find that for $\omega_{c} \gg \omega_{o}$, the dominant term is $G\left(\omega_{c}, \omega_{o}\right) \approx-\omega_{o}^{2} \ln \omega_{c}$. If $\omega_{o}$ is itself large the formula is

$$
G\left(\omega_{c}, \omega_{o}\right) \approx-\omega_{o}^{2} \ln \left(\frac{\omega_{c}}{\omega_{o}}\right)
$$

For small $\omega_{o}$ and $\omega_{c} \gg \omega_{o}$ the denominator in the log is modified, but the $-\omega_{o}^{2} \ln \omega_{c}$ behaviour always holds. The next to leading terms are independent of $\omega_{c}$, but dependent on $\omega_{o}$ and include a phase. Beyond that terms are suppressed by powers of $\omega_{c}$. We have checked these asymptotics numerically.

To summarize, the amplitude for emitting high energy closed strings, when the brane initial state has been perturbed by a reasonably energetic open string which is subsequently absorbed, is

$$
A_{2}\left(\omega_{c}, \omega_{o}\right) \approx-i \pi \frac{(2 \pi \lambda)^{-i\left(\omega_{c}+\omega_{o}\right)}}{\sinh \left(\pi\left(\omega_{c}+\omega_{o}\right)\right)}\left(\frac{\omega_{o}}{\omega_{c}}\right)^{\omega_{o}^{2}} \ldots
$$

where the ellipses represent terms that are either constant or fall off as $\omega_{c}$ increases.

For the unperturbed initial state of the D-brane (the spatially homogeneous decay), it was found in [8] that the total energy of closed strings emitted was divergent for a Dp brane with $p \leq 2$ (but see also [36]). We now examine how this statement is modified when the initial state is perturbed by addition of the boundary tachyon vertex operator. To compute the expectation value of total emitted energy we add up the squares of the individual emission amplitudes,

$$
\frac{E}{V_{p}} \sim \sum_{s} \frac{1}{2}\left|A_{2}^{(s)}\left(\omega_{c}, \omega_{o}\right)\right|^{2}
$$

For large $\omega_{c}$

$$
\left|A_{2}\left(\omega_{c}, \omega_{0}\right)\right| \sim e^{-2 \pi \omega_{c}} \omega_{c}^{-\omega_{0}^{2}}
$$

This differs from the unperturbed case in the extra factor of $\omega_{c}^{-\omega_{o}^{2}}$. A similar calculation to the one done in [8] yields

$$
\frac{E}{V_{p}} \sim \frac{1}{(2 \pi)^{p}} \int d \omega_{c} \omega_{c}^{-\frac{p}{2}-\omega_{o}^{2}} .
$$


Notice that the extra factor of $\omega_{c}^{-\omega_{o}^{2}}$ is a suppression if $\omega_{o}$ is real but an enhancement for imaginary $\omega_{o}$. Without the initial state perturbation, the average energy per unit volume diverges for the D0, D1, and D2 branes. For the D0 brane, the on shell condition fixes the initial state perturbation with the open-string tachyon vertex operator to be $e^{X^{0}}$, i.e. $\omega_{o}=i$ and the perturbation enhances the closed string emission leading to a divergent result. For the D1 brane, the result depends on the details of the perturbation. If the spatial momentum is large enough, such that $\omega_{0}>1$, the closed string emission will be finite. For higher dimensional branes, the total emission will be finite for any perturbation with real $\omega_{0}$. If the spatial momentum on any brane is sufficiently low, the on-shell condition will cause $\omega_{o}$ to be imaginary, and there will be an enhancement of closed string emission. For $p \leq 3$ the energy contained in closed string emission will diverge in this case.

\subsection{Comments}

For the D0 brane, the divergent closed string emission is expected to be an artifact of perturbation theory, which will be regulated by back reaction and quantum effects. It strongly suggests that the initial D-brane decays completely into closed strings and that tachyon matter, the end product of the decay in classical open string theory, is made up of very heavy closed strings [8]. A similar interpretation is given to the divergent emission of closed strings from the unperturbed D1 and D2 branes. For higher dimensional branes $(p \geq 3)$ the conventional wisdom has been that the decay process is more elaborate since it proceeds inhomogeneously ([6], [37], [38]). Notice, however, that in a quantum mechanical treatment of a decaying brane of any dimension, there will always be a component of the brane wavefunction along operators with imaginary $\omega_{o}$. This is because such an open string operator will be proportional to $e^{\left|\omega_{o}\right| X^{0}}$ and so be very small at early times, in effect corresponding to an infinitesimal perturbation of the initial state. We have shown that such perturbations enhance closed string emission for all Dp-branes, even when the decay is homogeneous, and would lead to divergent emission (without treating backreaction and wavefunction effects) for $p \leq 3$. In fact, we will get a divergent emission for any $p$ if we are willing to consider non-normalizable perturbations with imaginary spatial momenta. This enhancement of closed string emission is appealing. It suggests that a full treatment of decaying branes of any dimension will show all the energy of the brane entering into a state of heavy closed strings.

In our study of the open-closed amplitude we have discussed the open string state as an in-state, or a perturbation of the initial condition, while the closed string is emitted as an out-state. By complex conjugating we could have naively written down an amplitude that would apparently describe the emission of an open string. Likewise, at the perturbative level, we could have computed amplitudes containing additional strings in the final state including, apparently, open strings. This seems puzzling given that the brane has decayed at late times and thus open strings should not exist. It is possible that such amplitudes are simply inconsistent at a non-perturbative level. However, it seems more reasonable that we should interpret such amplitudes with "open strings" in the final state as follows. In any time dependent background we construct vertex operators at early and late times (in- and out- operators) to describe simple perturbations around the respective vacua. However, we are always free to use the complete basis of in-operators to describe outgoing states also 
- these operators will simply describe very complicated out-states. In our context, both open and closed strings can exist at early times, but only closed strings exist at late times. An out-open-string operator should thus represent some very complicated correlated state of closed strings that emerges from the open string via the decay of the brane. This interpretation leads to the important question of whether, in order to compute the full decay of a brane, we must calculate amplitudes with all possible out vertex operators, or whether computing the emission of closed strings as in [8], or as done above, suffices. In other words, do we include all decay channels that are perturbatively apparent including the ones with final-state "open strings", or is this "double counting"? The answer to this question goes to the heart of the open-closed duality for decaying branes that has been conjectured by Sen ([10], [14]). Regardless, the amplitudes computed above are essential components of the full answer and are the complete result for the "exclusive" amplitude describing absorption of the initial open string and emission of the final closed string.

\section{The bulk-bulk amplitude}

Now we turn to a computation of the bulk-bulk amplitude which describes the scattering of a closed string from the decaying brane, or the emission/absorption of a correlated pair of closed strings during the decay. To obtain the relevant correlator from (3.7) and (3.8) we use the conformal symmetry (but recall footnote 10) to locate one vertex operator at $z=0$ and the other at $z=r, 0 \leq r \leq 1$ on the unit disk. This gives

$$
A_{2}\left(\omega_{1}, \omega_{2}, r\right)=|r|^{-\omega_{1} \omega_{2}}\left(1-|r|^{2}\right)^{-\omega_{2}^{2} / 2} \int d x^{0} e^{i x^{0}\left(\omega_{1}+\omega_{2}\right)} F\left(0, \omega_{1}, r, \omega_{2} ; \mu\right)
$$

with $\mu=-x^{0}-\ln (-2 \pi \lambda)$ and

$$
\begin{aligned}
F\left(0, \omega_{1}, r, \omega_{2} ; \mu\right) & =\sum_{N=0}^{\infty} e^{-N \mu} J_{N}\left(\omega_{2}, r\right) \\
J_{N}\left(\omega_{2}, r\right) & =\int_{U(N)} d U|\operatorname{det}(1-r U)|^{2 i \omega_{2}} .
\end{aligned}
$$

To compute the full amplitude we should integrate over $r$ as $\int_{0}^{1} d r r$.

\subsection{The Toeplitz determinant}

The key steps in computing the amplitude are to evaluate the terms $J_{N}$ in the infinite series (5.2) and carry out the sum. Each $J_{N}$ is an expectation value of a periodic function with respect to the circular unitary ensemble (Appendix B):

$$
\begin{aligned}
J_{N}\left(\omega_{2}, r\right) & =\frac{1}{N !} \int_{-\pi}^{\pi} \prod_{a} \frac{d t_{a}}{2 \pi} \prod_{a=1}^{n}\left|1-r e^{-i t_{a}}\right|^{2 i \omega_{2}} \prod_{a<b}\left|e^{i t_{a}}-e^{i t_{b}}\right|^{2} \\
& \equiv \mathbf{E}_{N}\left\{\prod_{i=1}^{N} f\left(t_{i}\right)\right\} .
\end{aligned}
$$


By Heine's identity [39], this expectation value is equal to the Toeplitz determinant of the Fourier coefficients of $f$,

$$
\mathbf{E}_{N}\left\{\prod_{i=1}^{n} f\left(t_{i}\right)\right\}=D_{N}[f] \equiv \operatorname{det}\left(\hat{f}_{j-k}\right)_{1 \leq j, k \leq N}
$$

By this notation we mean the determinant of a matrix in which the entry in the $j^{\text {th }}$ row and $k^{\text {th }}$ column is the $(j-k)^{\text {th }}$ Fourier coefficient of $f$. For reference, the proof of this identity is included in the Appendix B. In our case the Fourier coefficients are (denoting $\left.\alpha=-i \omega_{2}\right)$

$$
\hat{f}_{k}=\hat{f}_{|k|}=\int \frac{d t}{2 \pi}\left|1-r e^{-i t}\right|^{-2 \alpha} e^{-i k t}=\frac{r^{|k|}}{|k| B(\alpha,|k|)} F\left(\alpha,|k|+\alpha,|k|+1 ; r^{2}\right) .
$$

Here $B$ is a beta function and $F$ is a hypergeometric function. This integral representation of the hypergeometric function is valid for $\alpha \neq 0,-1,-2 \cdots$ and $|r|<1$ [40]. As we will see below, the boundary limit $r \rightarrow 1$ of the right hand side will be well-defined anyway. The restriction on $\alpha$ can also be written as $\omega_{2} \neq 0,-i,-2 i,-3 i \cdots$.

- Special imaginary momenta: When $\omega_{2}=i, 2 i, 3 i \cdots$ (i.e., positive imaginary integer momenta), or equivalently $\alpha=1,2,3 \cdots$ with $\alpha \leq N$ we know that the integral (5.2) could have been done by the group theoretic methods of [16] with the result that

$$
J_{N}\left(\omega_{2}, r\right)=\left(1-r^{2}\right)^{\omega_{2}^{2}} \quad ; \quad \omega_{2}=i n, n \in Z^{+} \quad ; \quad n \leq N
$$

Since these momenta are within the range of validity of the integral representation (5.5), the determinant $D_{N}[f]$ in (5.4) must reproduce (5.6) at positive imaginary integer $\omega_{2}$. It is easy to verify that this is indeed the case, at least for small $n$ and $N$ but we have not constructed a general proof.

- Boundary limit gives Selberg: As $r^{2} \rightarrow 1$, the integrals and coefficients (5.4) reduce to (4.11) of the previous bulk-boundary calculation. In the limit, the determinants (5.4) reduce to

$$
D_{N}[f]_{r=1}=\left(\frac{\Gamma(1-2 \alpha)}{(\Gamma(1-\alpha)) \Gamma(\alpha)}\right)^{N} \operatorname{det}\left\{\frac{\Gamma(\alpha+|i-j|)}{\Gamma(1+|i-j|-\alpha)}\right\}_{i, j=1, \ldots, N} .
$$

Hence this determinant must be equal to (4.11):

$$
J_{N}\left(\omega_{2}, 1\right)=D_{N}[f]_{r=1}=\prod_{j=1}^{N} \frac{\Gamma(j) \Gamma(j-2 \alpha)}{(\Gamma(j-\alpha))^{2}}
$$

We have checked the identity explicitly with Maple for $N=1, \ldots, 8$, but have not constructed a general proof.

These two special limits appear initially to be in contradiction with each other since, for $\omega_{2}=i n$, (5.6) diverges as $r \rightarrow 1$, while (5.8) is finite. (Also see the discussion around 
(4.21).) However, what we really seeing here is that the order of limits can matter when one approaches the boundary at $r \rightarrow 1$ at special momenta. Our Toeplitz determinant (5.4) provides a definition of the two point amplitude for general momenta and gives a well-defined analytic continuation to the bulk-boundary amplitudes (with $r=1$ ) that we derived in the previous section. In addition the determinant reproduces the special form (5.6) which was previously obtained at imaginary integer moment [16]. Therefore, it appears that naive analytic continuation of (5.6) to general $\omega_{2}$, which is the technique used in the past, is invalid, as one might have expected.

Below we will see that the naive continuation of (5.6) from imaginary integers to general $\omega_{2}$ amounts to a large $\mathrm{N}$ approximation.

\subsection{Large $N$ approximation}

In general, dealing with the Toeplitz determinants is very complicated. We will consider two methods. For large $N$, approximate results can be found by using Szegö's limit theorem. This estimate can be improved by using an identity due to Borodin and Okounkov [41] (see also [42]). The latter authors considered Toeplitz determinants $D_{n}[f]$ of periodic functions and related them to Fredholm determinants of certain kernels. In particular, they considered functions of type

$$
f=\left(1+\xi_{1} \eta\right)^{z}\left(1+\xi_{2} \eta^{-1}\right)^{z^{\prime}}
$$

where $\xi_{1}, \xi_{2}, z, z^{\prime}$ are complex parameters and $\eta$ is a coordinate on a complex plane. If we set $\eta=-e^{-i t}, \xi_{1}=\xi_{2}=r$, and $z=z^{\prime}=-\alpha$, we recover our particular case. Here $\alpha$ can be an arbitrary complex number. Following [41], there is an identity

$$
D_{N}[f]=Z \operatorname{det}\left(1-K_{N}\right) .
$$

The prefactor $Z$ involves the Fourier coefficients of $\ln f$ :

$$
Z=\exp \left(\sum_{k=1}^{\infty} k(\widehat{\ln f})_{k}(\widehat{\ln f})_{-k}\right)
$$

This is the large $N$ approximation for the determinant given by Szegö's limit theorem, $\lim _{N \rightarrow \infty} D_{N}[f]=Z$, whereas the factor $\operatorname{det}\left(1-K_{N}\right)$ contains all the corrections to the approximation. To evaluate $Z$ we use the relation [40] (assuming $0 \leq r^{2} \leq 1$ )

$$
\widehat{(\ln f})_{k}=-\frac{\alpha}{2 \pi} \int_{-\pi}^{\pi} \ln \left(1-2 r \cos (t)+r^{2}\right) e^{-i k t} d t=-\alpha \frac{r^{|k|}}{|k|}
$$

which gives

$$
D_{N}[f] \approx Z=\exp \left\{\alpha^{2} \sum_{k=1}^{\infty} k r^{2 k} k^{-2}\right\}=\left(1-r^{2}\right)^{-\alpha^{2}}
$$

This large $N$ approximation reproduces the naive analytic continuation $n^{2} \rightarrow \alpha^{2}$ of (3.16) which results from calculations at imaginary integer momenta [16]. In fact, as we showed 
in (5.6), when $\alpha=n, n \in Z^{+}, n \leq N$, the result (5.13) is exact, or equivalently, the Borodin-Okounkov determinant $\operatorname{det}\left(1-K_{N}\right)$ equals 1 . There appears to be a remarkable localization phenomenon in the amplitude (5.2) such that for special momenta the result localizes to the large $N$ result $Z$ (5.11).

Notice that the right hand side of (5.13) is independent of the dimension $N$ of the matrix. It is dangerous to use this approximate large $N$ result in the bulk-bulk amplitude (5.2) because of the infinite sum, but if we do so regardless, we get (restoring $\alpha=-i \omega_{2}$ )

$$
F\left(0, \omega_{1}, r, \omega_{2} ; \mu\right)=\sum_{N=0}^{\infty} e^{-N \mu}\left(1-r^{2}\right)^{\omega_{2}^{2}}=\frac{\left(1-r^{2}\right)^{\omega_{2}^{2}}}{1+2 \pi \lambda e^{x^{0}}}
$$

Putting this back in the complete amplitude (5.1) and integrating over $x^{0}$ gives

$$
A_{2}\left(\omega_{1}, \omega_{2}, r\right)=-i \pi \frac{(2 \pi \lambda)^{-i\left(\omega_{1}+\omega_{2}\right)}}{\sinh \left(\pi\left(\omega_{1}+\omega_{2}\right)\right)}|r|^{-\omega_{1} \omega_{2}}\left(1-|r|^{2}\right)^{\omega_{2}^{2} / 2} .
$$

This gives the same result as in (3.18), computed by doing the zero mode integral first and using naive continuation from large imaginary integer momenta. The full amplitude is computed by including the spatial part of the amplitude and integrating over the position of the vertex operator. To do this recall that the spatial part of the conformal field is unchanged by the boundary perturbation, so we can include the contributions for a standard D-brane in (2.11), and write

$$
\mathbf{A}_{2}=\int_{0}^{1} d r r A_{2}\left(\omega_{1}, \omega_{2}, r\right)=-i \pi \frac{(2 \pi \lambda)^{-i\left(\omega_{1}+\omega_{2}\right)}}{\sinh \left(\pi\left(\omega_{1}+\omega_{2}\right)\right)} \int_{0}^{1} d r r|r|^{t}\left(1-|r|^{2}\right)^{s / 2-2+\omega_{2}^{2}}
$$

where we used the Mandelstam variables (2.2) and the closed string tachyon on-shell condition ((2.3) with $N=0)$. Finally, integrating over $r$ gives the result

$$
\mathbf{A}_{2}=-i \pi \frac{(2 \pi \lambda)^{-i\left(\omega_{1}+\omega_{2}\right)}}{\sinh \left(\pi\left(\omega_{1}+\omega_{2}\right)\right)} \frac{\Gamma((t / 2)+1) \Gamma\left(\vec{k}_{\|}^{2}-1\right)}{\Gamma\left((t / 2)+\vec{k}_{\|}^{2}\right)}
$$

where $\vec{k}_{||}$is the spacelike part of the momentum in the directions parallel to the brane. The factor $\Gamma(t / 2+1)$ in this expression contains the closed string poles (2.3). The remaining poles coming from the $\Gamma\left(\vec{k}_{\|}^{2}-1\right)$ are more exotic, since they occur at special spacelike momenta. If momentum here was not simply the spacelike piece, we would be recovering open string poles.

Above we pointed out that the boundary limit $r \rightarrow 1$ of the full amplitude (5.8) gives the result of the Selberg integral that we used to compute the bulk-boundary amplitudes in Sec. 4. By contrast, the large $N$ approximation described here, since it coincides with the naive continuation from imaginary integer momenta, gives a singular boundary limit. (See (4.21) and the discussion around it.) In Sec. 4.3 we argued that the singular behavior of the naive analytic continuation could be related to a need to regulate and renormalize bulk operators as they approach the boundary and collide with the boundary perturbation. 
Here we have seen that a large $N$ approximation of the bulk result leads to a singular boundary limit, while the full expression leads to the finite quantity that was used in Sec. 4 to study the effect of initial state perturbations on the decay of the brane. It may be useful to think of the large $N$ approximation (5.13) as a saddlepoint contribution, with the Borodin-Okounkov determinant in (5.10) containing corrections due to fluctuations in the interactions between the vertex operators and the boundary perturbations. Perhaps it is possible to understand the determinant in terms of a renormalization or dressing of string vertex operators by the boundary interaction.

Our result demonstrates that the usual technique for computing decaying brane amplitudes by analytically continuing from imaginary integer momenta is unreliable. The naive continuation only isolates a large $\mathrm{N}$ contribution to the full answer. This happens because of the remarkable localization of the full integral to the large $\mathrm{N}$ contribution at imaginary integer momenta. Our amplitudes are directly defined at real momenta and differ in important ways from the naive continuation. We have already discussed the consequences of the extra contributions for open-closed amplitudes. We now turn to a brief examination of the extra factor, the Borodin-Okounkov determinant, in a general amplitude.

\subsection{The Borodin-Okounkov determinant}

The determinant in the full amplitude $D_{N}[f]=Z \operatorname{det}\left(1-K_{N}\right)$ can be expressed (41] and [42]) as a Fredholm determinant of an operator $K_{N}$, acting on the $l_{2}$ normed infinite vector space $l_{2}(N, N+1, \ldots)$, defined by

$$
\operatorname{det}(1-K)=1+\sum_{m=1}^{\infty}(-1)^{m} \sum_{N \leq l_{1}<l_{2}<\cdots<l_{m}}^{\infty} \operatorname{det}\left[K\left(l_{i}, l_{j}\right)\right]_{i, j=1}^{m} .
$$

This equation is essentially a discrete version of the Fredholm determinant of a kernel familiar from the theory of integral equations. For the particular case of (5.9) (with the parameters replaced by ours), the kernel $K$ takes the form (correcting a minor error in 41])

$$
\begin{aligned}
K(i, j) & =\frac{(-\alpha)_{i+1}(-\alpha)_{j+1}}{i ! j !} r^{i+j+2}\left(1-r^{2}\right)^{-2 \alpha-1} \\
& \times \frac{1}{i-j}\left(F\left(\alpha,-\alpha, i+1, \frac{r^{2}}{r^{2}-1}\right) \frac{F\left(-\alpha-1,1+\alpha, j+2 ; \frac{r^{2}}{r^{2}-1}\right)}{j+1}\right. \\
& \left.-\frac{F\left(-1-\alpha, 1+\alpha, i+2 ; \frac{r^{2}}{r^{2}-1}\right)}{i+1} F\left(\alpha,-\alpha, j+1 ; \frac{r^{2}}{r^{2}-1}\right)\right) .
\end{aligned}
$$

The notation $(a)_{b}$ indicates Pochhammer symbols. From the point of view of the scattering amplitude, an important property of the kernel $K$ is that it contains the information that is missed in the naive analytic continuation of the previous results.

As a consistency check, we can examine the behavior of (5.18) as $r \rightarrow 0$. From (5.5) we can see that the Toeplitz determinants reduce to 1 as $r \rightarrow 0$. In addition, from (5.13) 
$Z \rightarrow 1$ as $r \rightarrow 1$, so we expect that $\operatorname{det}(1-K) \rightarrow 1$ as well. In the limit the kernel factorizes:

$$
K\left(l_{i}, l_{j}\right) \rightarrow r^{2} \cdot(-\alpha)_{l_{i}+1} r^{l_{i}}(-\alpha)_{l_{j}+1} r^{l_{j}} .
$$

As $r \rightarrow 0$ this of course implies that $\operatorname{det}(1-K) \rightarrow 1$. In fact, a stronger statement is true - $\operatorname{det} K$ vanishes to leading order in $r$ :

$$
\begin{aligned}
\operatorname{det}\left[K\left(l_{i}, l_{j}\right)\right]_{i, j=1}^{m} & =r^{2 m} \prod_{i=1}^{m}(-\alpha)_{l_{i}} r^{l_{i}} \\
& \times \sum_{\pi \in S_{m}}(-1)^{\pi}(-\alpha)_{l_{\pi(1)}} r^{l_{\pi(1)}} \cdots(-\alpha)_{l_{\pi(m)}} r^{l_{\pi(m)}}=0 .
\end{aligned}
$$

Because of the this the $\operatorname{det}(1-K)$ factor has no effect on the pole structure of the amplitude at $r=0$, in agreement with what we expect from the OPE of the tachyon vertex operators.

We already know that in the $r \rightarrow 1$ limit the Toeplitz determinant reduces to the form (5.8) and the bulk-bulk amplitude reduces to the bulk-boundary amplitude. Since the expression $Z$ (5.13) is singular as $r \rightarrow 1$, there must be a compensating singularity in $\operatorname{det}(1-K)$. This is more challenging to isolate. It would be very interesting to understand the properties of the determinant better since many aspects of the physics are clearly stored in it.

\subsection{What we learn}

We have given a prescription for defining the two point scattering amplitude for decaying branes at general momenta, and demonstrated that the usual tactic of naively continuing from imaginary integer momenta is a large $\mathrm{N}$ approximation that misses important contributions. The naive continuation fails because, remarkably, the general amplitude localizes to the large N contribution at these special momenta. Since we are using analytic continuation in our methods it is also possible that non-analytic contributions such as zero-momentum delta functions are missed. These are also essential to understand, because they make contributions to important quantities such as the stress tensor. In any case, it is clear that understanding the properties of Toeplitz determinants emerging from $\mathrm{U}(\mathrm{N})$ random matrix theory will lead to progress in the study of decaying branes and vice versa.

A useful way of making progress with the bulk-boundary amplitude in Sec. 4 was to do the integral over $x^{0}$ first, thus avoiding the need to carry out a perturbative sum over $N$ as in (5.2). Instead, the result then depends on a single $U(N)$ integral with $N$ being related to the total energy in the process. Such an approach might be useful here since for large energies the simple expression (5.13) might suffice.

The general amplitude prior to integrating over the time $x^{0}$ is:

$$
F\left(0, \omega_{1}, r, \omega_{2} ; \mu\right)=\sum_{N=0}^{\infty} e^{-N \mu} J_{N}\left(\omega_{2}, r\right) \quad ; \quad \mu=-x^{0}-\ln (-2 \pi \lambda) .
$$

This has the form of a computation in a grand canonical ensemble of $U(N)$ matrix models. At very early times $\left(x^{0} \rightarrow-\infty\right)$ the expression is dominated with smallest matrices. As 
$x^{0}$ increases, larger and larger matrices begin to play an important role. As $x^{0}$ increases beyond $-\ln (2 \pi \lambda)$ infinitely big matrices dominate the result and the formal power series fails to converge. To find the contribution to the amplitude from such late times, we must analytically continue the fully summed expression from convergent region $x^{0}<-\ln (2 \pi \lambda)$. In view of the many relationships between large $\mathrm{U}(\mathrm{N})$ matrices and closed strings, as well as recent developments in $2 \mathrm{~d}$ string theory ([1]], [12], [13], [14]), it is tempting to conclude that as the brane decays and spacetime becomes more closed-string-like in character, large $\mathrm{U}(\mathrm{N})$ matrices emerge from the dynamics of the disappearing open strings to describe the physical degrees of freedom of spacetime.

\section{Discussion}

Several interesting questions in the physics of decaying branes, including the nature of the final "tachyon matter" state and the possibility of defining a decoupling limit leading to timelike holography, require a deeper understanding of how strings scatter from such unstable objects. This has been a difficult problem to solve because the decay involves a non-trivial boundary interaction on the open string worldsheet. The standard technique to calculate amplitudes in this system is to first perform the necessary integrals at a special discrete set of momenta and then optimisitically "analytically continue" the resulting formal expressions. Of course this procedure is not strictly well-defined - an analytic function cannot be defined from its values at a discrete set of arguments without further information, such as either the behavior at infinity or consistency conditions derived from symmetry arguments. However, in the absence of further information this is the best we can do. In this paper we have made progress in arriving at a better-defined prescription for computing amplitudes.

First we showed that the timelike part of general amplitudes could be written in terms of correlators in $U(N)$ matrix models. (While we only studied tachyon scattering, vertex operators with more oscillators could be treated in a similar manner.) In a perturbative approach, a grand canonical ensemble of matrix models appeared, while integrating over the zero mode led to a single matrix expectation value. A key point is that the resulting open-closed 2-point amplitude can be exactly computed in an open subset of the complex momentum plane using Selberg's integral. Analytic continuation from this region to real momenta is reliable. Indeed, we were able to compute the open-closed amplitude in closed form in terms of zeta functions, and we derived the asymptotics of closed string emission when the initial condition for the decay is perturbed by an open string operator. In particular we found that for extended branes the emission of heavy closed strings could be enhanced or suppressed by the presence of the additional vertex operator, with interesting consequences for the structure of the "tachyon matter" state to which the brane decays. We likewise showed that the closed-closed scattering amplitude can be expressed exactly in terms of certain Toeplitz determinants of hypergeometric functions. The results obtained by naive analytic continuation from integer momenta amount to the leading large $N$ approximation (due to Szegö) of these determinants. In time dependent quantum field theories, different choices of analytic continuation in amplitudes are often related to different choices of vacuum state. We did not address the issue of the vacuum in detail here, but 
it would interesting to relate choices of analytic continuation to choices in the definition of the boundary state for the decaying brane. (See [8], [43], [19], [20], for some relevant discussion.)

In this paper we have focussed on the bulk-bulk and bulk-boundary two point functions, and it is an important problem to study more general correlation functions. For example, it would be interesting to study the boundary-boundary correlation function in depth using the ideas developed here. One would like to make contact with the Liouville based approach in, e.g., ([7], [18], [19], 20], 21]).

In addition to the techniques described in the main text there are other powerful tools that can be brought to bear on the subject of scattering from decaying branes. In Appendix $\mathrm{C}$ we show, by extending old techniques of Douglas [24], that there is a free fermion formulation of scattering from decaying branes. This is related to the free fermion techniques that can be used to study decaying branes in $2 \mathrm{~d}$ string theory ([11], [12], [13], [14]). Appendix D points out the relation between our scattering problem and a grand canonical ensemble for a classical Coulomb gas. It would be very interesting to apply these methods, and the techniques we have developed in the main text, to a study of tachyon matter and to explore potential decoupling limits leading to timelike holography, perhaps in the context of recently discovered non-singular spacetime backgrounds representing decaying branes (44, 45], 46], 47]).

Acknowledgments: We are grateful to Jan de Boer, Neil Constable, Eric D'Hoker, Michael Gutperle, Antti Kupiainen, and Volker Schomerus for useful conversations. Some of this work was carried out during the "Time and String Theory" workshop at the Aspen Center for Physics. V.B. was supported by the DOE under grant DE-FG02-95ER40893 and by the NSF under grant PHY-0331728. E.K-V. was supported in part by the Academy of Finland. P.K. was supported in part by NSF grant PHYT-0099590. A.N. was supported by Stiching FOM.

\section{Appendix A. Special momenta and summing over $N$ in the bulk-boundary amplitude}

At late (early) times $x^{0}$, the chemical potential $\mu$ (3.9), is large and negative (positive). Thus at late times, the series expression (4.5) combined with the Selberg result (4.11) for the bulk-boundary amplitude does not converge. To get an explicit function of $x^{0}$ we must sum (4.5) at early times and then analytically continue. Such a procedure is rather delicate - terms that are small at early times may dominate after the analytic continuation to late times, so the sum needs to be done exactly, or at least with attention paid to the pieces that dominate late time behavior. Progress can be made for special momenta.

We can write the amplitude (4.5) as

$$
F\left(0, \omega_{1}, 1, \omega_{2} ; \mu\right)=1+\sum_{N=1}^{\infty} x^{N} \prod_{j=1}^{N} \frac{\Gamma(j) \Gamma\left(j+2 i \omega_{2}\right)}{\left(\Gamma\left(j+i \omega_{2}\right)\right)^{2}}=1+\sum_{N=1}^{\infty} x^{N} c\left(N, \omega_{2}\right)
$$


with $x=e^{-\mu}=-2 \pi \lambda e^{x^{0}}$. The coefficients obey

$$
\frac{c\left(N+1, \omega_{2}\right)}{c\left(N, \omega_{2}\right)}=\frac{\Gamma(N+1) \Gamma\left(N+1+2 i \omega_{2}\right)}{\left(\Gamma\left(N+1+i \omega_{2}\right)\right)^{2}} .
$$

A series of this form is, by definition, a hypergeometric function if the ratio (A.2) is a rational function of $N$. In our case, this happens if $i \omega_{2}$ is a positive integer. In fact, when $i \omega_{2}=m$ is a non-negative integer a little algebra shows that $c(N,-i m)$ is a polynomial in $N$ :

$$
c(N,-i m)=\prod_{j=1}^{N} \frac{\Gamma(j) \Gamma(j+2 m)}{(\Gamma(j+m))^{2}}=\left(1+\frac{N}{m}\right)^{m} \prod_{k=1}^{m-1}\left[\left(1+\frac{N}{k}\right)\left(1+\frac{N}{2 m-k}\right)\right]^{k} .
$$

Furthermore $c(0,-i m)=1$.

Setting $i \omega_{2}=m$ the amplitude becomes $F(1,-i m ; \mu)=1+\sum_{N=1}^{\infty} x^{N} c(N,-i m)$ which we can be written

$$
F\left(0, \omega_{1}, 1, \omega_{2} ; \mu\right)=1+c\left(x \frac{d}{d x},-i m\right) \sum_{N=1}^{\infty} x^{N}=1+c\left(x \frac{d}{d x},-i m\right) \frac{x}{1-x}
$$

We thus arrive at

$$
F\left(0, \omega_{1}, 1,-i m ; \mu\right)=1+\left(1+\frac{x \frac{d}{d x}}{m}\right)^{m} \prod_{k=1}^{m-1}\left[\left(1+\frac{x \frac{d}{d x}}{k}\right)\left(1+\frac{x \frac{d}{d x}}{2 m-k}\right)\right]^{k} \frac{x}{1-x}
$$

The simplest cases are

$$
\begin{aligned}
F\left(0, \omega_{1}, 0,0 ; \mu\right) & =\frac{1}{1-x} \\
F\left(0, \omega_{1}, 1,-i ; \mu\right) & =\frac{1}{(1-x)^{2}} \\
F\left(0, \omega_{1}, 1,-2 i ; \mu\right) & =-\frac{1}{3} \frac{x^{3}-5 x^{2}+x-3}{(1-x)^{5}} .
\end{aligned}
$$

Note that the late time region is given by $\lim _{x_{0} \rightarrow \infty} x=-\infty$. It follows from (A.5) that

$$
\lim _{x_{0} \rightarrow \infty} F\left(0, \omega_{1}, 1,-i m ; \mu\right)=0 .
$$

So we find that for these special values of momenta the bulk-boundary amplitude vanishes at late time.

To summarize, (A.5) combined with (4.4) gives the result for the bulk-boundary twopoint function at special momenta. 


\section{Appendix B. Heine's Identity}

We consider integrals of the form

$$
I_{n}=\int \prod_{i=1}^{n} \frac{d t_{i}}{2 \pi} \prod_{i=1}^{n} f\left(t_{i}\right) \prod_{i<j}\left|e^{i t_{i}}-e^{i t_{j}}\right|^{2}
$$

containing a $2 \pi$-periodic function $f(t)$. In the Random matrix literature this corresponds to an expectation value of a function $\prod_{i=1}^{n} f\left(t_{i}\right)$ with respect to the circular unitary ensemble [32]. In our case, $f(t)=\left|1-z e^{-i t}\right|^{2 i k}$. A well known identity due to Heine [39], states that the expectation value of a periodic function $f(t)$ with respect to the circular unitary ensemble is equal to the Toeplitz determinant of the Fourier coefficients $\hat{f}_{k}=\int f(t) \exp (-i k t) d t$

$$
\frac{I_{n}}{n !}=D_{n}[f]=\operatorname{det}\left(\hat{f}_{(j-k)}\right)_{1 \leq j, k \leq n} .
$$

A Toeplitz determinant is a determinant of a (Toeplitz) matrix where all entries are equal along diagonals. For convenience, we review the proof of Heine's identity 39].

First, note that the $\prod_{i<j}\left|e^{i t_{i}}-e^{i t_{j}}\right|^{2}$ term is equal to $\Delta \bar{\Delta}$ where $\Delta$ is the Vandermonde determinant $\Delta=\operatorname{det}\left\{e^{i(k) t_{l}}\right\}_{k, l=1, \cdots n}$. The integral can be written as:

$$
I_{n}=\int \prod_{i=1}^{n} \frac{d t_{i}}{2 \pi} \prod_{i=1}^{n} f\left(t_{i}\right) \Delta \bar{\Delta} .
$$

Now expand the determinants out explicitly, $\Delta=\sum_{\Pi}(-1)^{\Pi} e^{i \sum_{l=1}^{n} \Pi(l) t_{l}}$, where $\Pi$ is a permutation of integers $1 \cdots n$. Hence,

$$
\Delta \bar{\Delta}=\sum_{\Pi_{1}, \Pi_{2}} \prod_{l=0}^{n-1}(-1)^{\Pi_{1}+\Pi_{2}} e^{i\left(\left(\Pi_{1}(l)-\Pi_{2}(l)\right) t_{l}\right)} .
$$

Inserting this in $(\mathbb{B} .3)$, we obtain

$$
I_{n}=\sum_{\Pi_{1}, \Pi_{2}}(-1)^{\Pi_{1}+\Pi_{2}} \prod_{l=1}^{n}\left\{\int \frac{d t_{l}}{2 \pi} f\left(t_{l}\right) e^{i\left[\left(\Pi_{1}(l)-\Pi_{2}(l)\right) t_{l}\right]}\right\} .
$$

Let $\nu_{l}=\Pi_{1}(l)-\Pi_{2}(l)$. Then

$$
I_{n}=\sum_{\Pi_{1}, \Pi_{2}}(-1)^{\Pi_{1}+\Pi_{2}} \prod_{l=1}^{n}\left\{\int \frac{d t_{l}}{2 \pi} f\left(t_{l}\right) e^{i \nu_{l} t_{l}}\right\} .
$$

We can fix the first permutation $\Pi_{1}=1,2,3, \cdots, n$ and multiply the result by $n$ !. This simplifies the above expression:

$$
\begin{aligned}
I_{n} & =n ! \sum_{\Pi}(-1)^{\Pi} \prod_{l=1}^{n}\left\{\hat{f}_{(l-\Pi(l))}\right\} \\
& =n ! \operatorname{det}\left(\hat{f}_{(l-m)}\right)_{1 \leq l, m \leq n} .
\end{aligned}
$$


The determinant in the final expression is a Toeplitz determinant of the Fourier coefficients of $f(t)$.

\section{Appendix C. Fermionization}

In this appendix, we outline a relation between the closed string scattering amplitudes and correlation functions in a theory of free fermions on a circle which gives an interesting reformulation of the scattering problem. The basic idea is due to Douglas [24] who showed the relation between $\mathrm{U}(\mathrm{N})$ group theory and a theory of $N$ free fermions on a circle. As we saw in section 3 , the scattering amplitudes can be written as a sum of correlators in a grand canonical ensemble of unitary matrix models, with time setting the fugacity:

$$
F\left(x_{0}, z, \omega_{c}\right)=\sum_{N=1}^{\infty}(-x)^{N} \int d M_{U(N)}|\operatorname{det}(1-z M)|^{2 i \omega_{c}}
$$

where $x=2 \pi \lambda e^{x_{0}}$. Using techniques developed in [24], we can show that this can be written as

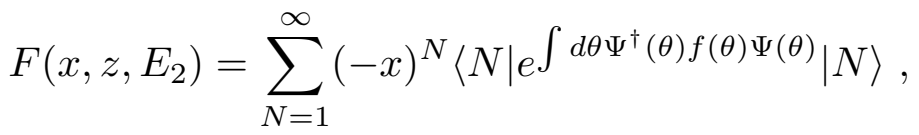

where $f(\theta)=-\mu \ln \left(1+r^{2}-2 r \cos \theta\right)$ and $|N\rangle$ is the $N$-fermion vacuum state satisfying

$$
\begin{aligned}
B_{n}|N\rangle & =0, \quad|n|>\frac{N-1}{2}, \\
B_{-n}^{\dagger}|N\rangle & =0, \quad|n| \leq \frac{N-1}{2} .
\end{aligned}
$$

Here, we have expanded the fermionic fields $\Psi(\theta)$ and $\Psi^{\dagger}(\theta)$ in terms of creation and annihilation operators as

$$
\Psi(\theta)=\sum_{n \in Z} B_{n} e^{i n \theta}, \quad \Psi^{\dagger}(\theta)=\sum_{n \in Z} B_{-n}^{\dagger} e^{-i n \theta}
$$

The expression in (C.2) is still quite cumbersome because we have to sum over expectation values in the $N$ fermion ground state. To obtain a more useful expression, we will add a factor of $e^{-\beta H}$ and sum over all states in the Hilbert space. Then, by projecting onto the $N$ fermion sector and taking the $\beta \rightarrow \infty$ limit correctly reproduces the sum over $N$ fermion ground states. Thus the sum in (C.2) can be written as the $\beta \rightarrow \infty$ limit of the following partition function:

$$
Z(\beta)=\operatorname{Tr}\left(e^{-\beta H} e^{\int d \theta \Psi^{\dagger}(\theta) f(\theta) \Psi(\theta)}(-x)^{N} P_{N}\right)
$$

where $P_{N}$ is the projection operator onto the $N$ particle sector:

$$
P_{N}=\int d \alpha e^{i \alpha(\hat{N}-N)}
$$


Here, $\hat{N}=\int d \theta \Psi^{\dagger} \Psi$ counts the number of fermions. The Hamiltonian $H$ in the $N$ fermion sector is given by

$$
H \equiv H_{0}+E_{0}=\frac{1}{2} \int d \theta \frac{d \Psi^{\dagger}}{d \theta} \frac{d \Psi}{d \theta}-\frac{N\left(N^{2}-1\right)}{12}
$$

where we have defined $H_{0}=\frac{1}{2} \int d \theta \frac{d \Psi^{\dagger}}{d \theta} \frac{d \Psi}{d \theta}$ and $E_{0}=-\frac{N\left(N^{2}-1\right)}{12}$. Notice that the $N$ fermion ground state $|N\rangle$ has zero energy. Using these definitions we can write (C.5) as

$$
Z(\beta)=\int d \alpha \operatorname{Tr}\left(e^{-\beta H_{0}} e^{\int d \theta \Psi^{\dagger}(\theta)(f(\theta)+i \alpha) \Psi(\theta)}\right) \sum_{N} e^{-i(\alpha+\pi) N+\beta E_{0}+\ln x}
$$

To proceed further, we notice that the trace in this expression can be computed by path integral methods.:

$$
\operatorname{Tr}\left(e^{-\beta H} O\right)=\int_{\Psi(0)=\Psi(\beta)} \mathcal{D} \Psi^{\dagger} \mathcal{D} \Psi e^{-S} O
$$

The path integral naturally computes the expectation values of operators which are normal ordered, which here means that all the $\Psi^{\dagger}$ 's are pushed to the left. Thus to compute the trace

$$
\operatorname{Tr}\left(e^{-\beta H_{0}} e^{\int d \theta \Psi^{\dagger}(\theta)(f(\theta)+i \alpha) \Psi(\theta)}\right)
$$

from a path integral, we write the operator $e^{\int d \theta \Psi^{\dagger}(\theta)(f(\theta)+i \alpha) \Psi(\theta)}$ as a normal ordered operator:

$$
e^{\int d \theta \Psi^{\dagger}(\theta)(f(\theta)+i \alpha) \Psi(\theta)}=: e^{\int d \theta \Psi^{\dagger}(\theta) e^{(f(\theta)+i \alpha)} \Psi(\theta)}: \text {. }
$$

Then, the trace can be written as a path integral expression as follows:

$$
\begin{aligned}
\operatorname{Tr}\left(e^{-\beta H_{0}} e^{\int d \theta \Psi^{\dagger}(\theta)(f(\theta)+i \alpha) \Psi(\theta)}\right) & =\int \mathcal{D} \Psi^{\dagger} \mathcal{D} \Psi e^{\int d \theta d t \Psi^{\dagger}\left(i \frac{\partial}{\partial t}+\frac{1}{2} \frac{\partial^{2}}{\partial \theta^{2}}+e^{(f(\theta)+i \alpha)} \delta(t)\right) \Psi} \\
& =\operatorname{det}\left(i \frac{\partial}{\partial t}+\frac{1}{2} \frac{\partial^{2}}{\partial \theta^{2}}+e^{i \alpha}\left(1+r^{2}-2 r \cos \theta\right)^{-\mu} \delta(t)\right)
\end{aligned}
$$

where the path integral is performed over periodic $\Psi$ 's satisfying $\Psi(0, \theta)=\Psi(\beta, \theta)$. This determinant is still non-trivial to compute and here we do not push it any further. If this determinant is computed, then the $\beta \rightarrow \infty$ limit of $Z(\beta)$ in (C.8) can give the amplitude (C.1).

\section{Appendix D. Relation to a grand canonical ensemble of a classical gas}

It is interesting to note that the series (5.2) can also be rewritten as the grand canonical partition function of a Coulomb gas of point charges in two dimensions, confined in a circle 
and interacting with two external point charges via repulsive Coulomb interactions [48]. The point charges on the circle interact via a two body potential

$$
V\left(t_{i}, t_{j}\right)=-\ln \left|e^{i t_{i}}-e^{i t_{j}}\right|
$$

The vertex operators at $z_{1}=0$ and $z_{2}=r$ correspond to two external point charges at $1 / z_{1}^{*}=\infty$ and $1 / z_{2}^{*}=1 / r$ of strength $-\alpha$, acting on the charges on the circle by the potential

$$
V\left(t_{j}\right)=\alpha \sum_{a=1,2} \ln \left|1-z_{a} e^{i t_{j}}\right|=\alpha \ln \left|1-r e^{i t_{j}}\right|
$$

Now, we can introduce a temperature $1 / \beta=1 / 2$, and the coefficients $J_{n}$ (5.3) can be interpreted as the partition function of a gas of $n$ charges interacting with the two external ones at temperature $T=1 / 2$ :

$$
Z_{N}(T)=J_{N}=\prod_{a} \int \frac{d t_{a}}{2 \pi} e^{-\beta\left(\sum_{a=1}^{n} V\left(t_{a}\right)+\sum_{a<b} V\left(t_{a}, t_{b}\right)\right)}
$$

If we formally introduce a fugacity $z=e^{\mu}=-2 \pi \lambda e^{x^{0}}$, the infinite series (5.2) becomes the grand canonical partition function:

$$
Q(T, z)=F\left(r, \alpha ; x^{0}\right)=\sum_{N=0}^{\infty} z^{N} Z_{N}(T)
$$

Note that for $\lambda>0$, the fugacity has a "wrong" negative sign. Further, eventually we will want $\alpha=i \omega_{2}$, so that the strength of the charge at $z_{2}=1 / r$ becomes imaginary. 


\section{References}

[1] A. Strominger, "The dS/CFT correspondence," JHEP 0110, 034 (2001) arXiv:hepth/0106113]; "Inflation and the dS/CFT correspondence," JHEP 0111, 049 (2001) arXiv:hep-th/0110087.

[2] V. Balasubramanian, J. de Boer and D. Minic, "Mass, entropy and holography in asymptotically de Sitter spaces," Phys. Rev. D 65, 123508 (2002) arXiv:hepth/0110108; "Holography, time and quantum mechanics," arXiv:gr-qc/0211003; "Exploring De Sitter Space And Holography," Class. Quant. Grav. 19, 5655 (2002) [Annals Phys. 303, 59 (2003)] arXiv:hep-th/0207245.

[3] M. Gutperle and A. Strominger, "Spacelike branes," JHEP 0204, 018 (2002) arXiv:hep-th/0202210.

[4] A. Sen, "Rolling tachyon," JHEP 0204, 048 (2002) arXiv:hep-th/0203211; A. Sen, "Tachyon matter," JHEP 0207, 065 (2002) arXiv:hep-th/0203265; A. Sen,

"Field theory of tachyon matter," Mod. Phys. Lett. A 17, 1797 (2002) arXiv:hepth/0204143].

[5] A. Strominger, "Open string creation by S-branes," arXiv:hep-th/0209090.

[6] F. Larsen, A. Naqvi and S. Terashima, "Rolling tachyons and decaying branes," JHEP 0302, 039 (2003) arXiv:hep-th/0212248.

[7] M. Gutperle and A. Strominger, "Timelike boundary Liouville theory," Phys. Rev. D 67, 126002 (2003) arXiv:hep-th/0301038.

[8] N. Lambert, H. Liu and J. Maldacena, "Closed strings from decaying D-branes," arXiv:hep-th/0303139.

[9] A. Sen, "Open and closed strings from unstable D-branes," Phys. Rev. D 68, 106003 (2003) arXiv:hep-th/0305011.

[10] A. Sen, "Open-closed duality at tree level," Phys. Rev. Lett. 91, 181601 (2003) arXiv:hep-th/0306137.

[11] J. McGreevy and H. Verlinde, "Strings from tachyons: The c $=1$ matrix reloaded," JHEP 0312, 054 (2003) arXiv:hep-th/0304224.

[12] I. R. Klebanov, J. Maldacena and N. Seiberg, "D-brane decay in two-dimensional string theory," JHEP 0307, 045 (2003) arXiv:hep-th/0305159.

[13] M. R. Douglas, I. R. Klebanov, D. Kutasov, J. Maldacena, E. Martinec and N. Seiberg, "A new hat for the $\mathrm{c}=1$ matrix model," arXiv:hep-th/0307195.

[14] A. Sen, "Open-closed duality: Lessons from matrix model," arXiv:hep-th/0308068.

[15] M. Goulian and M. Li, "Correlation Functions In Liouville Theory," Phys. Rev. Lett. 66, 2051 (1991).

[16] N. R. Constable and F. Larsen, "The rolling tachyon as a matrix model," JHEP 0306, 017 (2003) arXiv:hep-th/0305177. 
[17] J. Teschner, "Liouville theory revisited," Class. Quant. Grav. 18, R153 (2001) arXiv:hep-th/0104158.

[18] A. Strominger and T. Takayanagi, "Correlators in timelike bulk Liouville theory," Adv. Theor. Math. Phys. 7, 369 (2003) arXiv:hep-th/0303221.

[19] V. Schomerus, "Rolling tachyons from Liouville theory," JHEP 0311, 043 (2003) arXiv:hep-th/0306026.

[20] S. Fredenhagen and V. Schomerus, "On minisuperspace models of S-branes," JHEP 0312, 003 (2003) arXiv:hep-th/0308205.

[21] S. Fredenhagen and V. Schomerus, "Exact Rolling Tachyons", to appear.

[22] D. Gaiotto, N. Itzhaki and L. Rastelli, "Closed strings as imaginary D-branes," Nucl. Phys. B 688, 70 (2004) arXiv:hep-th/0304192.

[23] O. Bergman and S. S. Razamat, "Imaginary time D-branes to all orders," arXiv:hepth/0402124.

[24] M. R. Douglas, "Conformal field theory techniques for large N group theory," arXiv:hep-th/9303159.

[25] J. Polchinski, "String Theory", Cambridge University Press, 1998

[26] S. Hwang, "Cosets as gauge slices in SU(1,1) strings," Phys. Lett. B 276, 451 (1992) arXiv:hep-th/9110039; J. M. Evans, M. R. Gaberdiel and M. J. Perry, "The noghost theorem for $\operatorname{AdS}(3)$ and the stringy exclusion principle," Nucl. Phys. B 535, 152 (1998) arXiv:hep-th/9806024.

[27] T. Okuda and S. Sugimoto, "Coupling of rolling tachyon to closed strings," Nucl. Phys. B 647, 101 (2002) arXiv:hep-th/0208196].

[28] K. Okuyama, "Comments on half S-branes," JHEP 0309, 053 (2003) arXiv:hepth/0308172].

[29] J. de Boer, A. Sinkovics, E. Verlinde and J. T. Yee, "String interactions in c $=1$ matrix model," arXiv:hep-th/0312135.

[30] A. Sen, "Rolling tachyon boundary state, conserved charges and two dimensional string theory," arXiv:hep-th/0402157.

[31] P. Kraus, A. Ryzhov and M. Shigemori, "Strings in noncompact spacetimes: Boundary terms and conserved charges," Phys. Rev. D 66, 106001 (2002) arXiv:hepth/0206080.

[32] M. Mehta, "Random Matrices", Academic Press, London, second edition, 1991.

[33] J.P. Keating and N.C. Snaith, "Random Matrix Theory and $\zeta(1 / 2+i t)$ ", Commun. Math. Phys. 214, 57 (2000).

[34] A. Erdelyi, ed., "Higher Transcendental Functions, Vol. 1-3", Kreiger (Malabar, Florida) 1981.

[35] Eric W. Weisstein et al. "Hurwitz Zeta Function." From MathWorld-A Wolfram Web Resource. http://mathworld.wolfram.com/HurwitzZetaFunction.htm]; Also see http://functions.wolfram.com/ZetaFunctionsandPolylogarithms/Zeta2/06/02/. 
[36] J. L. Karczmarek, H. Liu, J. Maldacena and A. Strominger, "UV finite brane decay," JHEP 0311, 042 (2003) [arXiv:hep-th/0306132].

[37] A. Sen, "Time evolution in open string theory," JHEP 0210, 003 (2002) arXiv:hepth/0207105.

[38] P. Mukhopadhyay and A. Sen, "Decay of unstable D-branes with electric field," JHEP 0211, 047 (2002) arXiv:hep-th/0208142.

[39] H. Heine, "Kugelfunktionen", Berlin, 1878 and 1881. Reprinted Physica Verlag, Würzburg, 1961; G. Szegö, "Orthogonal Polynomials", AMS, New York, 1959.

[40] I. S. Gradshteyn and I. M. Ryzhik, "Table of Integrals, Series, and Products", corrected and enlarged edition, Academic Press 1980.

[41] A. Borodin and A. Okounkov, "A Fredholm determinant formula for Toeplitz determinants," Int. Eqs. Oper. Th. 37 (2000) 386-396 arXiv:math.CA/9907165.

[42] E. L. Basor and H. Widom, "On a Toeplitz determinant identity of Borodin and Okounkov," Int. Eqs. Oper. Th. 37 (2000) 397-401 arXiv:math.FA/9909010.

[43] A. Maloney, A. Strominger and X. Yin, "S-brane thermodynamics," JHEP 0310, 048 (2003) arXiv:hep-th/0302146.

[44] G. Jones, A. Maloney and A. Strominger, "Non-singular solutions for S-branes," arXiv:hep-th/0403050.

[45] J. E. Wang, "Twisting S-branes," arXiv:hep-th/0403094.

[46] G. Tasinato, I. Zavala, C. P. Burgess and F. Quevedo, "Regular S-Brane Backgrounds," arXiv:hep-th/0403156.

[47] H. Lu, C. N. Pope and J. F. Vazquez-Poritz, "From AdS black holes to supersymmetric flux-branes," arXiv:hep-th/0307001; H. Lu and J. F. Vazquez-Poritz, "Fourdimensional Einstein Yang-Mills de Sitter gravity from eleven dimensions," Phys. Lett. B 597, 394 (2004) arXiv:hep-th/0308104; H. Lu and J. F. Vazquez-Poritz, "Nonsingular twisted S-branes from rotating branes," JHEP 0407, 050 (2004) arXiv:hepth/0403248].

[48] T. H. Baker, P. J. Forrester and P. A. Pearce, "Random matrix ensembles with an effective extensive external charge", arXiv:cond-mat/9803355]. 